\title{
Objective medication adherence and persistence in people with multiple sclerosis: a systematic review, meta-analysis, and meta-regression
}

Joshua Mardan, BPsychSc; Mohammad Akhtar Hussain, MBBS, MD, FAFPHM, PhD; Michelle Allan, BN, MS Clinical Nursing, MS (NP); and Lisa B Grech, PhD, MPsych, MAPS

\section{What is already known about this subject}

- Suboptimal medication adherence occurs in approximately $50 \%$ of people who require regular medication administration across chronic diseases and is linked with poorer health outcomes and increased healthcare utilization.

- People with multiple sclerosis may be prescribed disease-modifying therapy, which is administered through self-injectable, oral, or intravenous administration routes; injectable and oral administration routes are associated with suboptimal adherence and persistence.

\section{What this study adds}

- Medication adherence (12-month comparison) was significantly higher for oral compared to injectable diseasemodifying therapies, with no difference in discontinuation rates for oral and injectable disease-modifying therapies.

- Medication adherence remained suboptimal for both oral and injectable disease-modifying therapies despite higher adherence with oral medication, which highlights the ongoing need for medication compliance initiatives.

- Differences in study methodologies, including measurement methods, definitions, and observation lengths, limited the comparative analysis that could be undertaken. The limitations highlight that future research would benefit from protocol consistency.

\section{Author affiliations}

Joshua Mardan, BPsychSc, School of Health Sciences, Swinburne University of Technology, and Department of Medicine, School of Clinical Sciences at Monash Health, Monash University, Melbourne, Australia. Mohammad Akhtar Hussain, MBBS, MD, FAFPHM, PhD, Public Health Unit, Central Coast Local Health District, New South Wales, Australia, and Menzies Institute for Medical Research, University of Tasmania, Hobart, Australia. Michelle Allan, BN, MS Clinical Nursing, MN (NP), Department of Neurology, Monash Health, Melbourne, Australia. Lisa B Grech, PhD, MPsych, MAPS, School of Health Sciences, Swinburne University of Technology; Department of Medicine, School of Clinical Sciences at Monash Health, Monash University; Department of Cancer Experiences Research, Peter MacCallum Cancer Centre; and Melbourne School of Psychological Sciences, University of Melbourne, Melbourne, Australia.

\section{AUTHOR CORRESPONDENCE: \\ Lisa B Grech, lisa.grech@monash.edu, +61 410.947.444}




\section{ABSTRACT}

BACKGROUND: Medication adherence is critical for the realization of pharmacotherapy benefits and reduced healthcare expenditure. Studies have shown up to $60 \%$ of people with Multiple sclerosis (MS) experience suboptimal medication adherence, which is associated with poorer health outcomes and subsequent discontinuation. The current systematic review reported on objectively measured adherence and discontinuation rates for self-administered oral and injectable disease-modifying therapies (DMTs).

OBJECTIVES: To identify whether, in people with MS, the introduction of oral DMTs has improved medication adherence when compared with injectable DMTs. The secondary aim was to report synthesized objectively measured medication adherence and persistence rates for both oral and injectable DMTs in MS across varying study durations.

METHODS: Literature searches were conducted through PubMed, Web of Science, Scopus, and PsycINFO. Inclusion criteria were limited to English, peer-reviewed, objective, self-administered DMT articles, published between July 1993 to December 2019. Publications reporting combined intravenous and self-administered DMT data, or that did not account for DMT switching in discontinuation rates, were excluded. Data were synthesized into observation lengths ranging from less than 8 months to greater than 36 months. Meta-analysis and meta-regression were undertaken on both oral and injectable 12-month adherence and discontinuation data.

RESULTS: In total, 61 articles were included; 46 articles examined adherence and 26 examined discontinuation. Twelve-month adherence ranged between $53.0 \%$ to $89.2 \%$ for oral $(\mathrm{N}=7)$ and $47.0 \%$ to $77.4 \%$ for injectable DMTs ( $N=7)$. Results from the meta-analysis and meta-regression show significantly higher pooled mean medication possession ratio (MPR) adherence for oral DMTs (91.0\%) when compared to injectable DMTs (77.0\%) over 12 months ( $\beta=-0.146 ; 95 \% \mathrm{Cl}$ : -0.263 to -0.029 ). Results indicate major asymmetry across studies (LFK index: -5.18), proposing the presence of significant publication bias. Mean discontinuation over 12 months was between $10.5 \%$ to $33.3 \%$ for oral $(\mathrm{N}=7)$ and $15.2 \%$ to $50.8 \%$ for injectable DMTs $(\mathrm{N}=10)$, with meta-analysis results indicating the presence of significant heterogeneity (I2 Injectable: $99.5 \%$; 12 Oral: $93.1 \%$ ) between studies included in each subgroup. However, no appreciable difference in mean discontinuation rates across groups (Injectable: $27 \%$; $95 \%$ Cl: $19.0 \%-34.0 \%$; Oral: $24 \%$; $95 \% \mathrm{Cl}$ : $17.0 \%-31.0 \%$ ) was found.

CONCLUSIONS: Medication adherence for oral DMTs suggests a significant improvement compared to adherence for injectable DMTs. No significant difference in discontinuation rates between oral and injectable DMTs was found. Oral DMT adherence and persistence studies are limited, given their relatively recent introduction. Suboptimal medication adherence and discontinuation issues remain present for both oral and injectable DMTs. Future studies would benefit from improved consistency in methodology, such as comparable adherence and persistence definitions.
Multiple sclerosis (MS) is a chronic, neurodegenerative disease with a reported $10.4 \%$ increase in global prevalence between 1990 and 2016, to over 2.2 million cases. ${ }^{1,2}$ While there is no cure for MS, disease-modifying therapies (DMTs), ${ }^{3,4}$ reduce annualized relapse rates by $18.0 \%$ to $66.0 \%{ }^{5,6}$ and disability progression by $12.0 \%$ to $41.0 \%{ }^{6}$

Optimal medication adherence is crucial to maximize drug efficacy and reduce unnecessary healthcare expenditure. However, World Health Organization ${ }^{7}$ data estimates approxmimately $50 \%$ of people with chronic diseases experience suboptimal medication adherence. Medication adherence in MS is no exception. Adherence rates for selfinjectable DMTs are reported as between $41 \%$ to $88 \%$ across varying measurement types, ${ }^{8}$ and between $30 \%$ to $60 \%$ of people with MS discontinue self-injectable DMTs within two years. ${ }^{9-11}$

The introduction of oral DMTs has not resolved the problem. A study assessing one-year adherence and persistence to oral DMTs-fingolimod (FTY), teriflunomide (TFN), and dimethyl fumarate (DMF)-reported that the adherence rate, measured by proportion of days covered (PDC), was less than $80 \%$ in $24.6 \%$ to $46.9 \%$ of participants with discontinuation rates between $25.6 \%$ to $50.3 \%$. $^{12}$ Studies consistently showed an increased likelihood of suboptimal medication adherence or discontinuation with increasing treatment durations. ${ }^{13-15}$

Medication adherence is "The extent to which a patient acts in accordance with the prescribed interval, and dose of a dosing regimen." ${ }^{16}$ Persistence is "The duration of time from initiation to discontinuation of therapy." ${ }^{16}$ Discontinuation is the cessation of therapy, which may be initiated by either the clinician or patient. ${ }^{17}$ Suboptimal medication adherence may be intentional or unintentional. Intentional suboptimal adherence has been linked to perceived inefficacy, ${ }^{18}$ cost,$^{19}$ and medication side effects. Side effects vary by DMT, but they may include fatigue, headache, nausea, or diarrhea. Specific to route of administration, injectable DMTs may incur injection site reactions and needle phobia. ${ }^{3}$ Forgetting is the most common unintentional reason for suboptimal medication adherence. Factors such as physical disability, cognitive impairment, and depression-all common in MS patients-contribute to medication adherence difficulties. ${ }^{3,18}$ Medication adherence may be assessed objectively, using, for example, pharmacy records, or via patient self-report.

Two previous systematic reviews have been undertaken addressing medication adherence and persistence to selfadministered injectable DMTs in people with MS, ${ }^{8,15}$ both of which predated studies that assessed medication adherence and persistence in oral DMTs. Two recent reviews of oral DMT adherence and discontinuation rates did not compare oral and injectable DMTs to quantify whether oral 
DMTs show improved compliance. ${ }^{20,21}$ Given the changed landscape of DMT options, our primary aim was to identify whether oral DMTs, when compared with injectable DMTs, have increased medication adherence and persistence to self-administered DMTs in people with MS. Secondarily, we synthesized objectively measured medication adherence and persistence rates for self-administered oral and injectable DMTs in MS across varying study durations.

\section{Methods}

\section{SEARCH STRATEGY}

Literature searches were conducted through PubMed, Web of Science, Scopus, PsycINFO, CINHAL (via EBSCOhost), and the Cochrane Library. Key publication reference lists were also searched. The search included articles published from the first approved DMT, on July 23, 1993, ${ }^{22}$ through December 31, 2019. The search strategy included all US Food and Drug Administration approved MS DMTs to capture studies reporting both intravenously (IV) and self-administered DMTs, although IV administered DMT data were excluded.

Search terms included: multiple sclerosis, medication adherence, adherence, compliance, concordance, persistence, disease-modifying therapy, disease-modifying treatments, oral medication, treatment, therapy, therapeutics, and drug therapy, as well as each of the DMT generic and brand names. Six search strings were constructed from the search terms incorporating truncations (eg, diseasemodifying therap*).

\section{STUDY SELECTION}

Inclusion was limited to peer-reviewed articles, in English, assessing medication adherence and persistence for all self-administered oral or injectable DMTs, in adults with MS ( $\geq 18$ years; any subtype) taken on a regular basis ( $\geq$ once every two weeks). Studies that did not explicitly account for switching methods were excluded. We additionally excluded conference proceedings abstracts.

\section{DATA EXTRACTION}

Abstracts were screened for relevance by two independent investigators (JM, LG), who mutually agreed upon differences. Inter-rater reliability was calculated. Following full-text screening, data were extracted including patients, study, measurement instrument characteristics, adherence and/or persistence/discontinuation results, correlations between adherence/discontinuation, and differences between groups. Extracted adherence values included mean medication possession ratio (MPR) and proportion of days covered (PDC), binary adherence cut-off score, reported at least $80 \%$ adherence unless otherwise specified, or effect sizes (ES) and standard errors. Where unavailable, the standard errors were calculated from the standard deviation and sample size. The MPR is the sum of the days' supply for all fills of a given drug in a particular time period, divided by the number of days in the time period. The PDC is the ratio of the number of days covered by the medication in a period, divided by the total number of days in the period. Studies that reported adherence using medication event monitoring systems (MEMS) bottle caps and electronic autoinjectors typically utilized the MPR calculation: medication administered divided by expected medication. Adherence measures that differed from the above were reported according to the specific calculation.

\section{DATA SYNTHESIS AND ANALYSIS}

We synthesized adherence and discontinuation studies into four observation lengths, ranging from less than 8 months to greater than 36 months (Supplementary Table 1, 2, 3, available in online article). Where applicable, adherence values for two or more injectable or oral DMTs reported within a study were calculated to form a single injectable or oral value utilizing an online calculator . Only baseline (preintervention) data are reported for intervention studies.

\section{EFFECT SIZE POOLING}

Due to an appropriate number of comparative studies, meta-analysis and meta-regression were undertaken for 12-month adherence and 12-month discontinuation. ES for studies were pooled using the inverse variance heterogeneity (IVhet) meta-analysis model, ${ }^{23}$ which uses a quasi-likelihood model based variance structure without distributional assumptions and has coverage probabilities for $95 \%$ confidence intervals (CI). This model has outperformed the random effect (RE) method and is superior to conventional RE and/or fixed-effect models for inter-study heterogeneity, ${ }^{23,24}$ as the IVhet model retains correct coverage probabilities and lower observed variance. ${ }^{23}$

Heterogeneity and consistency were evaluated with Cochran's Q and the I2 statistics, respectively. Practically significant heterogeneity was indicated (I2>50\%). Asymmetry between meta-analysis studies was examined graphically using doi plot and the Luis Furuya-Kanamori (LFK) index, ${ }^{25}$ as the doi plot represents an improvement over the classic funnel plot, while the LFK index outperforms Egger's regression P-value for detection of asymmetry. ${ }^{25}$ Both doi plot and LFK indicated major asymmetry between studies, indicating heterogeneity between studies from type, combinations of DMTs, methods adopted, study population heterogeneity, or publication bias. As a result, overall pooled estimates for comparing oral vs injectable DMTs were not calculated. Instead, an IVhet meta-regression 


\section{FIGURE 1 PRISMA Flow Chart}

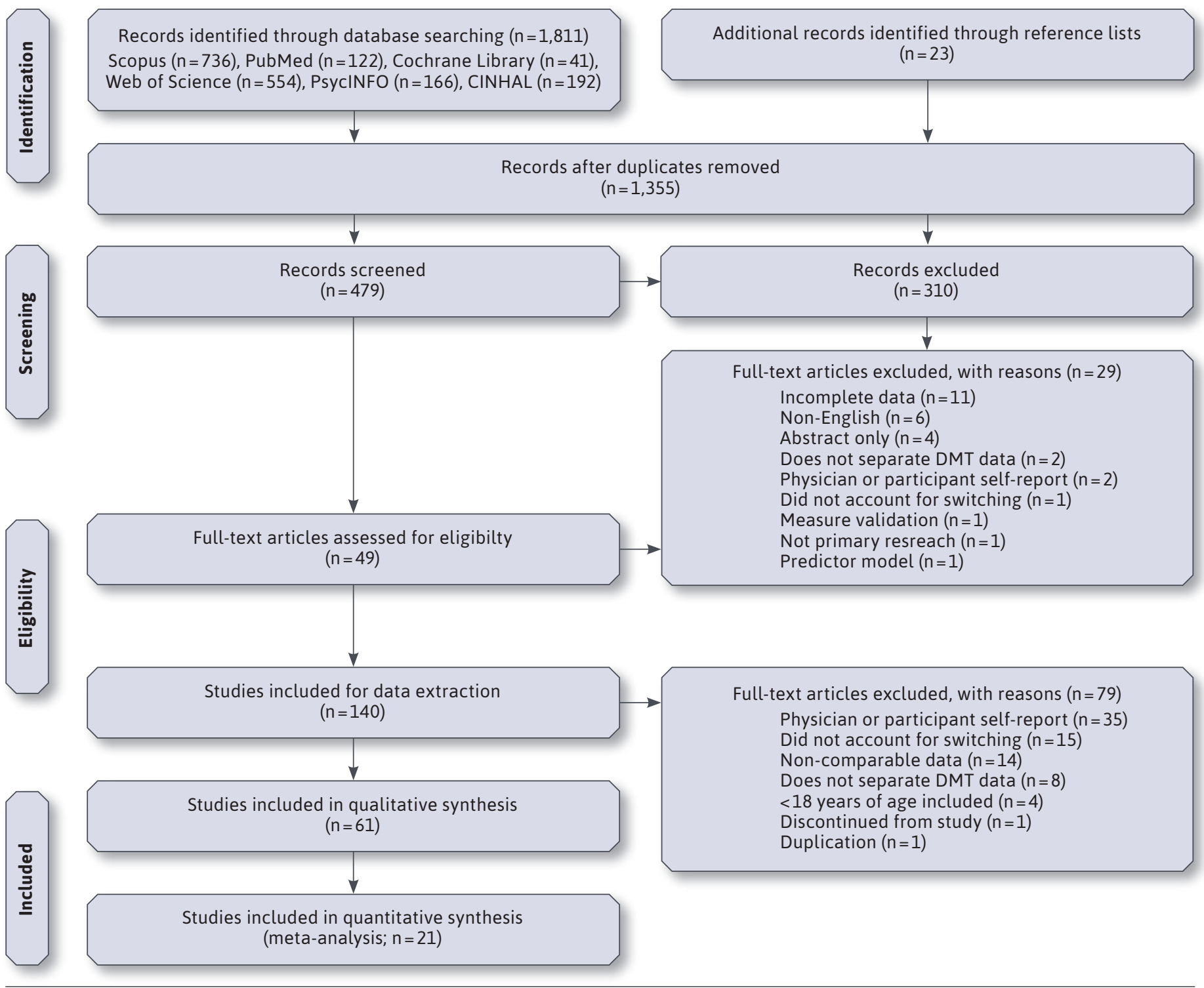

Notes: PRISMA Flowchart of search strategy, with the reasons for exclusion.

Adapted from Moher D, Liberati A, Tetzlaff J, Altman DG, Group P. Preferred reporting items for systematic reviews and meta-analyses: the PRISMA statement. BMJ 2009;339(7716):b2535.

model to examine the heterogeneity in the meta-analysis was used. ${ }^{26}$ The Huber-Eicker-White-sandwich error variances generated correct standard errors in metaregression, equivalent to the IVhet model in meta-analysis. For the 12-month adherence meta-regression, studies were divided into two subgroups: oral DMTs (Group-1, N=6) and injectable DMTs (Group-2, N=8).
Discontinuation studies included in this review reported different discontinuation definitions, including continuous discontinuation of medication for more than 30 days, more than 60 days, more than 90 days, or any discontinuation. Due to limited studies that reported these measures in each group, we clubbed them together irrespective of discontinuation definition. Discontinuation studies were 
analysed in two subgroups: oral DMTs (Group-1, eight data sources; $\mathrm{N}=7$ studies) and injectable DMTs (Group-2, 11 data sources; $\mathrm{N}=10$ studies).

\section{SENSITIVITY ANALYSIS}

Given high heterogeneity between the studies, we performed sensitivity analyses to explore the effect of excluding individual studies on the overall pooled estimates and level of heterogeneity. The analyses showed no discernible changes (Supplementary Table 5, available in online article).

Meta-analyses were carried out using MetaXL version 5.3. Meta-regression was performed in Stata.

\section{APPRAISAL TOOL FOR CROSS-SECTIONAL STUDIES (AXIS)}

Study reporting quality was assessed using the appraisal tool for cross-sectional studies (AXIS), ${ }^{27}$ undertaken by one author (JM). The assessment was cross-checked by a second author (LG).

\section{ETHICS, PROTOCOL REGISTRATION AND REPORTING}

This secondary data analysis did not require ethics approval and informed consent. The systematic review was registered with the International Prospective Register of Systematic Reviews (PROSPERO; CRD42019122412) and reported per the Preferred Reporting Items for Systematic Reviews and Meta-Analysis (PRISMA) guidelines. ${ }^{28}$

\section{Results}

\section{SEARCH SUMMARY}

Database searches yielded 1,811 publications (Scopus: 736, PubMed: 122, Cochrane Library: 41, Web of Science: 554, PsychINFO: 166, and CINHAL: 192; Figure 1). Twenty-three additional records were identified from key reference lists. A total of 1,355 duplicates were removed, leaving 479 abstracts screened against inclusion criteria. Inter-rater reliability for abstract inclusion was substantial $(\kappa=0.785$, $\mathrm{P}<0.001){ }^{29}$ Following mutual adjudication of 46 records, 140 studies underwent full-text screening, and 79 articles were excluded. In total, 38 studies were excluded because they only measured self-reported adherence and/or discontinuation. The remaining 61 studies underwent data extraction and were included in the qualitative synthesis. Forty-six studies examined adherence, and 26 studied examined discontinuation. Twenty-one studies were included in the quantitative analysis.

\section{STUDY CHARACTERISTICS}

Study characteristics are displayed in Table 1. Twenty-six studies were conducted in the United States, 10 in Canada, seven in Italy, three each in Germany, France and Spain, two in Switzerland, and one each in Greece, Sweden, Denmark, as well as one international multisite and two European multisite studies. Forty-four studies were retrospective, 16 prospective, and one had retrospective and prospective observation periods. Data of included studies were primarily collected from database claims $(\mathrm{N}=22)$, multicentre or clinic $(\mathrm{N}=19)$, database $(\mathrm{N}=9)$, administrative $(\mathrm{N}=6)$, or clinical data $(\mathrm{N}=5)$. Twelve studies examined both injectable and oral DMTs, 40 examined injectable DMTs only, and nine studies examined oral DMTs only. From these studies, 12 examined adherence of oral DMTs and 40 examined the adherence of injectable DMTs. Twenty-six studies examined DMT discontinuation ( $\mathrm{N}=13$ oral; $\mathrm{N}=21$ injectable).

Twenty-four studies included participants with relapse remitting MS (RRMS); four included RRMS and secondary progressive MS (SPMS); two included RRMS, SPMS, and primary progressive MS (PPMS); one included RRMS and clinically isolated syndrome (CIS); and one included RRMS, SPMS, and CIS participants. Twenty-nine studies did not report MS subtype. Overall, observation lengths ranged between eight weeks to 18 years, although the majority of studies assessed adherence over 12 -month $(\mathrm{N}=24)$ and 24 -month $(\mathrm{N}=13)$ periods. Study sample size ranged between 30 to 50,057 participants. Mean participant age across studies $(\mathrm{N}=54)$ ranged between 32.3 to 55.0 years.

\section{BIAS ASSESSMENT}

Quality of reporting, assessed with the AXIS ${ }^{27}$ (Supplementary Table 4, available in online article), showed studies were clear in their statement of aims. The overall study methodology was appropriate in design, description, and sample size justification. A notable issue with US studies, which could potentially limit generalizability, was the use of insurance claims databases for 20 (76.9\%) of 26 studies. One study recruited veterans ${ }^{30}$ with a primarily male sample and older mean age than other studies. Ethics approval and consent were obtained for 17 (27.9\%) studies, with ethics only obtained for 15 (24.6\%), and consent only obtained for six (9.8\%) studies. Ethics approval and/or consent was exempt by local laws for 12 (19.7\%) studies, while there was no explicit comment about consent or ethics attainment for $11(18.0 \%)$ studies. Study results were adequately described in general. However, as secondary study outcomes, the results were sometimes limited in adherence and/or discontinuation data. Discussions were largely an appropriate reflection of study results, with most studies discussing limitations, albeit some were brief. Generalizability issues were sparsely mentioned. The studies were predominantly funded by pharmaceutical companies $(\mathrm{N}=35 ; 57.4 \%)$. The authors received honorariums, speaker, consultancy fees or 


\section{TABLE 1 Characteristics of Included Objective Studies}

\begin{tabular}{|c|c|c|c|c|c|c|c|c|}
\hline $\begin{array}{l}\text { Author, } \\
\text { year }\end{array}$ & Country & $\begin{array}{l}\text { Study } \\
\text { type }\end{array}$ & $\begin{array}{c}\text { Observation } \\
\text { length }\end{array}$ & Sample size & $\begin{array}{l}\text { Age, gender, subtype, } \\
\text { MS duration, EDSS }\end{array}$ & Design/setting & DMT(s) & Comments \\
\hline $\begin{array}{l}\text { Agashivala, } \\
2013^{41}\end{array}$ & USA & $\mathrm{AH}, \mathrm{DC}$ & 12 months & $N=1,891$ & $\begin{array}{l}\text { Age: } M=45.6, S D=11.1 \\
\text { years; Gender: } 76.4 \% \\
\text { Female; Subtype: NR; } \\
\text { MS duration: NR; EDSS: } \\
\text { NR }\end{array}$ & $\begin{array}{l}\text { Retrospective/ } \\
\text { database } \\
\text { claims }\end{array}$ & $\begin{array}{l}\text { FTY, IFN } \beta-1 b \text {, } \\
\text { IFN } \beta-1 a, G A\end{array}$ & $\begin{array}{l}\text { New oral } \\
\text { DMT users }\end{array}$ \\
\hline $\begin{array}{l}\text { Aungst, } \\
2019^{42}\end{array}$ & USA & $\mathrm{AH}$ & 52 weeks & $\begin{array}{c}\mathrm{N}=35 \\
\text { (consented); } \\
\mathrm{N}=25 \text { (ana- } \\
\text { lyzed) }\end{array}$ & $\begin{array}{l}\text { Age: } M=41.1, S D=11.6 \\
\text { years; Gender: } 71.0 \% \\
\text { Female; Subtype: NR; } \\
\text { MS duration: NR; EDSS: } \\
\text { NR }\end{array}$ & $\begin{array}{l}\text { Prospective/ } \\
\text { multicentre }\end{array}$ & DMF & \\
\hline $\begin{array}{l}\text { Bayas, } \\
2015^{37}\end{array}$ & $\begin{array}{l}\text { Fifteen } \\
\text { European } \\
\text { Countries }\end{array}$ & $\mathrm{AH}$ & 12 months & $\begin{array}{c}\mathrm{N}=912 \\
\text { (safety } \\
\text { population); } \\
\mathrm{N}=791 \\
\text { (completed) }\end{array}$ & $\begin{array}{l}\text { Age: } M=36.3, S D=10.3 \\
\text { years; Gender: } 73.7 \% \\
\text { Female; Subtype: } \\
\text { RRMS; } M S \text { duration: } \\
\text { Analyzed subset: } \\
N=710, M=33.0 \\
S D=49.8 \text { months; } \\
\text { EDSS: } M=1.8, S D=1.4\end{array}$ & $\begin{array}{l}\text { Prospective/ } \\
\text { multicentre }\end{array}$ & IFN $\beta$-1a s.c & $\begin{array}{l}\text { Commenced } \\
\text { IFN } \beta-1 a<8 \\
\text { weeks prior } \\
\text { to study } \\
\text { enrollment }\end{array}$ \\
\hline $\begin{array}{l}\text { Bonafede, } \\
2013^{39}\end{array}$ & USA & DC & 24 months & $N=5,710$ & $\begin{array}{l}\text { Age: } M=44.6, S D=10.4 \\
\text { years; Gender: } 76.6 \% \\
\text { Female; Subtype: NR; } \\
\text { MS duration: NR; EDSS: } \\
\text { NR }\end{array}$ & $\begin{array}{l}\text { Retrospective/ } \\
\text { database } \\
\text { claims }\end{array}$ & $\begin{array}{l}\text { IFN } \beta-1 a \text {, } \\
\text { IFN } \beta-1 b, G A \text {, } \\
\text { FTY }\end{array}$ & \\
\hline $\begin{array}{l}\text { Braune, } \\
2016^{43}\end{array}$ & Germany & DC & $\begin{array}{c}24 \text { months; } \\
\text { PM: } M=1037.8 \\
(461.2) \text { days }\end{array}$ & $\begin{array}{c}\text { N=433; PM: } \\
\text { N=198 } \\
\text { (99 FTY, } \\
99 \text { iDMT) }\end{array}$ & $\begin{array}{l}\text { Age: } M=40.1, S D=9.8 \\
\text { years (PM only); } \\
\text { Gender: } 75.8 \% \text { Female; } \\
\text { Subtype: RRMS; } M S \\
\text { duration: } M=7.4 \\
S D=5.9 \text { years; EDSS: } \\
M=2.3, S D=1.5\end{array}$ & $\begin{array}{l}\text { Retrospective/ } \\
\text { outpatient } \\
\text { clinical data }\end{array}$ & $\begin{array}{l}\text { IFN } \beta-1 a \text {, } \\
\text { IFN } \beta-1 b, G A \text {, } \\
\text { FTY }\end{array}$ & $\begin{array}{l}\text { 1st injectable } \\
\text { failed and } \\
\text { switched to } \\
\text { fingolimod or } \\
\text { 2nd injectable. } \\
\text { Analysis of } 1 \\
\text { and } 2 \text { years for } \\
\text { persistence; } \\
\text { Propensity } \\
\text { matched sam- } \\
\text { ple persistence } \\
\text { and discontinu- } \\
\text { ation across } \\
\text { available } \\
\text { observation } \\
\text { days }\end{array}$ \\
\hline $\begin{array}{l}\text { Bruce, } \\
2010^{14}\end{array}$ & USA & $\mathrm{AH}$ & 8 weeks & $N=67$ & $\begin{array}{l}\text { Age: } M=46.2, S D=10.6 \\
\text { years; Gender: } 88.1 \% \\
\text { Female; Subtype: } \\
\text { RRMS; MS duration: } \\
M=10.0, S D=7.8 \text { years; } \\
\text { EDSS: } M=2.4, S D=1.2\end{array}$ & $\begin{array}{l}\text { Prospective/ } \\
\text { MS specialty } \\
\text { clinic }\end{array}$ & $\begin{array}{l}\text { GA, IFN } \beta-1 a \text {, } \\
\text { IFN } \beta-1 b\end{array}$ & \\
\hline $\begin{array}{l}\text { Burks, } \\
2017^{13}\end{array}$ & USA & $\mathrm{AH}, \mathrm{DC}$ & 12 months & $N=1,018$ & $\begin{array}{l}\text { Age: } 18-24(4.3 \%), \\
\text { 25-44 (14.2\%), 35-44 } \\
\text { (30.6\%), 45-54 (32.0\%), } \\
\text { 55-64 (19.0\%); Gender: } \\
\text { 72.1\% Female; } \\
\text { Subtype: NR; MS } \\
\text { duration: NR; EDSS: NR }\end{array}$ & $\begin{array}{l}\text { Retrospective/ } \\
\text { database } \\
\text { claims }\end{array}$ & TFN, FTY, DMF & $\begin{array}{l}\text { Injectable } \\
\text { data combined } \\
\text { intravenous, } \\
\text { thus, were } \\
\text { excluded }\end{array}$ \\
\hline
\end{tabular}




\section{TABLE 1 Characteristics of Included Objective Studies (continued)}

\begin{tabular}{|c|c|c|c|c|c|c|c|c|}
\hline $\begin{array}{l}\text { Author, } \\
\text { year }\end{array}$ & Country & $\begin{array}{l}\text { Study } \\
\text { type }\end{array}$ & $\begin{array}{l}\text { Observation } \\
\text { length }\end{array}$ & Sample size & $\begin{array}{l}\text { Age, gender, subtype, } \\
\text { MS duration, EDSS }\end{array}$ & Design/setting & DMT(s) & Comments \\
\hline $\begin{array}{l}\text { Cerghet, } \\
2010^{44}\end{array}$ & USA & $\mathrm{AH}$ & 12 months & $\begin{array}{c}\mathrm{N}=214 \\
\text { (eligible); } \\
\mathrm{N}=163 \\
\text { (completed); } \\
\mathrm{N}=111 \\
\text { (dispensed a } \\
\text { DMT) }\end{array}$ & $\begin{array}{l}\text { Age: } M=47.5, S D=9.5 \\
\text { years; Gender: } 81 \% \\
\text { Female; Subtype: } \\
\text { RRMS; } M S \text { duration: } \\
M=8.9, S D=6.4 \text { years; } \\
\text { EDSS: } M=4.9\end{array}$ & $\begin{array}{l}\text { Retrospective/ } \\
\text { database } \\
\text { claims }\end{array}$ & $\begin{array}{l}\text { GA, IFN } \beta-1 a \text {, } \\
\text { IFN } \beta-1 b\end{array}$ & \\
\hline $\begin{array}{l}\text { Cohan, } \\
2018^{45}\end{array}$ & USA & $\mathrm{AH}$ & 12 months & $\begin{array}{c}N=708 ; N=531 \\
\text { (no switch); } \\
N=177 \text { (switch) }\end{array}$ & $\begin{array}{l}\text { Age: No switch: } \\
M=47.5, S D=9.9 \text { years. } \\
\text { Switch: } M=46.8 \\
\text { SD }=9.6 \text { years; Gender: } \\
78.5 \% \text { Female (Switch } \\
\text { and no switch groups); } \\
\text { Subtype: NR; MS dura- } \\
\text { tion: NR; EDSS: NR }\end{array}$ & $\begin{array}{l}\text { Retrospective/ } \\
\text { database } \\
\text { claims }\end{array}$ & $\begin{array}{l}\text { IFN } \beta-1 a \text {, } \\
\text { IFN } \beta-1 b\end{array}$ & $\begin{array}{l}\text { Participants } \\
\text { who switched } \\
\text { DMTs versus } \\
\text { those who } \\
\text { remain con- } \\
\text { sistent on one } \\
\text { DMT for } 12 \\
\text { months }\end{array}$ \\
\hline $\begin{array}{l}\text { Cohen, } \\
2015^{46}\end{array}$ & USA & $\mathrm{AH}$ & 24 months & $\begin{array}{c}\mathrm{N}=3,151 \\
\text { (enrolled); } \\
\mathrm{N}=2,966 \\
\text { (baseline); } \\
\mathrm{N}=2,410 \\
\text { (completed) }\end{array}$ & $\begin{array}{l}\text { Age: } M=49.6, S D=9.9 \\
\text { years; Gender: } 80.9 \% \\
\text { Female; Subtype: } \\
\text { RRMS, SPMS, PPMS; } \\
\text { MS duration: } M=9.6 \\
\text { SD =8.3 years; EDSS: } \\
M=3.0, \text { SD }=2.5\end{array}$ & $\begin{array}{l}\text { Prospective/ } \\
\text { specialty } \\
\text { pharmacy }\end{array}$ & $\begin{array}{l}\text { GA, IFN } \beta-1 a \text {, } \\
\text { IFN } \beta-1 b\end{array}$ & \\
\hline $\begin{array}{l}\text { Condé, } \\
2019^{47}\end{array}$ & France & DC & 24 months & $N=346$ & $\begin{array}{l}\text { Age: } M=44.2, S D=0.9 \\
\text { years; Gender: } 80.3 \% \\
\text { Female; Subtype: } \\
\text { RRMS; MS duration: } \\
M=10.1, S D=0.8 \text { years; } \\
\text { EDSS: } M=1.8, S D=0.1\end{array}$ & $\begin{array}{l}\text { Retrospective/ } \\
\text { clinical record }\end{array}$ & DMF, TFN & \\
\hline $\begin{array}{l}\text { D'Amico, } \\
2019^{48}\end{array}$ & Italy & DC & 24 months & $\begin{array}{c}\mathrm{N}=903 \\
\text { pwRRMS; } \\
\mathrm{N}=316(\mathrm{TFN}) \\
\mathrm{N}=587(\mathrm{DMF})\end{array}$ & $\begin{array}{l}\text { Age: } M=42.5, S D=10.6 \\
\text { years; Gender: } 67.5 \% \\
\text { Female; Subtype: } \\
\text { RRMS; MS duration: } \\
\text { M=109.2, SD = 84.6 } \\
\text { months; EDSS: TFN: } \\
2.0(1.5-3.5) ; \text { DMF: } 1.5 \\
(1.0-3.0)\end{array}$ & $\begin{array}{l}\text { Retrospective/ } \\
\text { multicentre }\end{array}$ & DMF, TFN & \\
\hline $\begin{array}{l}\text { Defer, } \\
2018^{49}\end{array}$ & France & $\mathrm{AH}$ & $M=11.5$ years & $N=15,039$ & $\begin{array}{l}\text { Age: NR; Gender: } \\
\text { 73.9\% Female; } \\
\text { Subtype: RRMS, SPMS; } \\
\text { MS duration: } M=11.5 \text {, } \\
\text { SD = } 9.7 \text { years; EDSS: } \\
\text { NR }\end{array}$ & $\begin{array}{l}\text { Retrospective/ } \\
\text { multicentre }\end{array}$ & $\begin{array}{l}\text { IFN } \beta-1 a \text {, } \\
\text { IFN } \beta-1 b, G A\end{array}$ & $\begin{array}{l}\text { Only examines } \\
\text { first-line } \\
\text { injectable } \\
\text { DMTs } \\
\text { adherence }\end{array}$ \\
\hline $\begin{array}{l}\text { Deftereos, } \\
2018^{50}\end{array}$ & Greece & $\mathrm{AH}$ & 12 months & $\begin{array}{l}\mathrm{N}=64 \\
\text { (enrolled); } \\
\mathrm{N}=47 \\
\text { (complete } \\
\text { study) }\end{array}$ & $\begin{array}{l}\text { Age: } M=36.2, S D=11.2 \\
\text { years; Gender: } 78.1 \% \\
\text { Females; Subtype: } \\
\text { RRMS; MS duration: } \\
\text { M=2.1, SD = 3.0 years; } \\
\text { EDSS: NR }\end{array}$ & $\begin{array}{l}\text { Prospective/ } \\
\text { multicentre }\end{array}$ & IFN $\beta$-1a s.c & \\
\hline $\begin{array}{l}\text { Degil } \\
\text { Esposti, } \\
2017^{40}\end{array}$ & Italy & $\mathrm{AH}, \mathrm{DC}$ & 36 months & $N=1,698$ & $\begin{array}{l}\text { Age: } M=40.0, S D=10.7 \\
\text { years; Gender: } 69.0 \% \\
\text { Female; Subtype: } N R \text {; } \\
\text { MS duration: } M=5.9 \\
S D=6.3 \text { years; EDSS: } \\
\text { Range, } 0-9\end{array}$ & $\begin{array}{l}\text { Retrospective/ } \\
\text { administrative } \\
\text { database }\end{array}$ & $\begin{array}{l}\text { IFN } \beta-1 a \text {, } \\
\text { IFN } \beta-1 b, G A\end{array}$ & \\
\hline
\end{tabular}




\section{TABLE 1 Characteristics of Included Objective Studies (continued)}

\begin{tabular}{|c|c|c|c|c|c|c|c|c|}
\hline $\begin{array}{l}\text { Author, } \\
\text { year }\end{array}$ & Country & $\begin{array}{l}\text { Study } \\
\text { type }\end{array}$ & $\begin{array}{c}\text { Observation } \\
\text { length }\end{array}$ & Sample size & $\begin{array}{l}\text { Age, gender, subtype, } \\
\text { MS duration, EDSS }\end{array}$ & Design/setting & DMT(s) & Comments \\
\hline $\begin{array}{l}\text { Devonshire, } \\
2016^{51}\end{array}$ & Canada & $\mathrm{AH}$ & $\begin{array}{l}12 \text { and } 24 \\
\text { weeks }\end{array}$ & $\begin{array}{c}\mathrm{N}=162 \\
\text { (enrolled); } \\
\mathrm{N}=158 \\
\text { (analyzed) }\end{array}$ & $\begin{array}{l}\text { Age: } M=37.4, S D=9.8 \\
\text { years; Gender: } 75.3 \% \\
\text { Female; Subtype: } \\
\text { RRMS, SPMS; } M S \\
\text { duration: } M=24.0 \text {, } \\
\text { SD }=57.6 \text { months; } \\
\text { EDSS: } M=1.8, S D=1.3 .\end{array}$ & $\begin{array}{l}\text { Prospective/ } \\
\text { multicentre }\end{array}$ & IFN $\beta$-1a s.c. & \\
\hline $\begin{array}{l}\text { Duquette, } \\
2019^{9}\end{array}$ & Canada & $\mathrm{AH}, \mathrm{DC}$ & 24 months & $\begin{array}{c}\mathrm{N}=26,157 \\
\text { (compliance); } \\
\mathrm{N}=32,795 \text { (dis- } \\
\text { continuation) }\end{array}$ & $\begin{array}{l}\text { Age: NR; Gender: NR; } \\
\text { Subtype: NR; MS } \\
\text { duration: NR; EDSS: NR }\end{array}$ & $\begin{array}{l}\text { Retrospective/ } \\
\text { database } \\
\text { claims }\end{array}$ & $\begin{array}{l}\text { IFN } \beta \text {-1a, } \\
\text { IFN } \beta-1 b, \text { GA, } \\
\text { FTY, DMF, TFN }\end{array}$ & $\begin{array}{l}\text { Demographics } \\
\text { not reported }\end{array}$ \\
\hline $\begin{array}{l}\text { Eriksson, } \\
2018^{52}\end{array}$ & Sweden & $D C$ & $\begin{array}{c}\text { Median }=2.5 \\
\text { years }\end{array}$ & $N=400$ & $\begin{array}{l}\text { Age: DMF 1st: } M=35.3, \\
\text { SD=9.8. DMF switch: } \\
\text { M=40.5, SD=10.1; } \\
\text { Gender: DMF 1st: } \\
\text { 72.0\% Female. DMF } \\
\text { switch: } 76.0 \% \text { Female; } \\
\text { Subtype: NR; MS dura- } \\
\text { tion: NR; EDSS: NR }\end{array}$ & $\begin{array}{l}\text { Retrospective/ } \\
\text { regional } \\
\text { database }\end{array}$ & DMF & $\begin{array}{l}\text { Compares DMF } \\
\text { as 1st line and } \\
\text { switch to DMF }\end{array}$ \\
\hline $\begin{array}{l}\text { Ernst, } \\
2017^{53}\end{array}$ & USA & DC & 24 months & $N=450$ & $\begin{array}{l}\text { Age: } M=45.5, S D=12.1 \\
\text { years; Gender: } 75.8 \% \\
\text { Female; Subtype: } \\
\text { RRMS; MS duration: } \\
M=527.6, S D=1600.5 \\
\text { days; EDSS: NR }\end{array}$ & $\begin{array}{l}\text { Retrospective/ } \\
\text { administrative } \\
\text { data }\end{array}$ & $\begin{array}{l}\text { IFN } \beta \text {-1a s.c., } \\
\text { DMF }\end{array}$ & \\
\hline $\begin{array}{l}\text { Evans, } \\
2017^{54} \\
2016^{55}\end{array}$ & Canada & $\mathrm{AH}, \mathrm{DC}$ & $\begin{array}{c}\text { 12-month } \\
\text { adherence; } \\
\mathrm{M}=7.8 \\
\mathrm{SD}=4.0 \text { years } \\
\text { discontinuation } \\
\text { follow-up }\end{array}$ & $\begin{array}{c}N=4,746 \\
(2017) ; \\
N=4,830 \\
(2016)\end{array}$ & $\begin{array}{l}\text { Age: } M=40.4, S D=10.0 \\
\text { years; Gender: } 75.8 \% \\
\text { Female; Subtype: NR; } \\
\text { MS duration: NR; EDSS: } \\
\text { NR }\end{array}$ & $\begin{array}{l}\text { Retrospective/ } \\
\text { administrative } \\
\text { data }\end{array}$ & $\begin{array}{l}\text { IFN } \beta-1 a \text {, } \\
\text { IFN } \beta-1 b, G A\end{array}$ & $\begin{array}{l}\text { Duplicate } \\
\text { studies, both } \\
\text { included as } \\
\text { one reports } \\
\text { MPR (2017) } \\
\text { and one PDC } \\
\text { (2016) }\end{array}$ \\
\hline $\begin{array}{l}\text { Evans, } \\
2012^{56}\end{array}$ & Canada & DC & Up to 14 years & $N=1,896$ & $\begin{array}{l}\text { Age: } M=40.2, S D=9.5 \\
\text { years; Gender: } 75.1 \% \\
\text { Female; Subtype: } \\
\text { RRMS, SPMS; MS } \\
\text { duration: NR; EDSS: } \\
\text { Median, } 2.5 \text { (range, } \\
0-8.5 \text { ) }\end{array}$ & $\begin{array}{l}\text { Retrospective/ } \\
\text { MS database }\end{array}$ & $\begin{array}{l}\text { IFN } \beta-1 a \text {, } \\
\text { IFN } \beta-1 b, G A\end{array}$ & \\
\hline $\begin{array}{l}\text { Fernández, } \\
2016^{57}\end{array}$ & Spain & $\mathrm{AH}$ & Up to 3 years & $N=258$ & $\begin{array}{l}\text { Age: } M=40.7, S D=9.5 \\
\text { years; Gender: } 67.8 \% \\
\text { Female; Subtype: } \\
\text { RRMS; } M S \text { duration: } \\
M=8.9, S D=6.0 \text { years; } \\
\text { EDSS: } M=1.8, S D=1.2\end{array}$ & $\begin{array}{l}\text { Retrospective/ } \\
\text { multicentre }\end{array}$ & IFN $\beta$-1a s.c. & \\
\hline $\begin{array}{l}\text { Fernández- } \\
\text { Fournier, } \\
2015^{58}\end{array}$ & Spain & DC & $\begin{array}{l}\text { Median }=34 \\
\text { months }\end{array}$ & $N=155$ & $\begin{array}{l}\text { Age: } M=37.0, S D=9.0 \\
\text { years; Gender: } 68.3 \% \\
\text { Female; Subtype: } \\
\text { RRMS; MS duration: } \\
\text { NR; EDSS: Median =1.5- } \\
2, \text { IQR }=1-3\end{array}$ & $\begin{array}{l}\text { Retrospective/ } \\
\text { clinical } \\
\text { records }\end{array}$ & GA & $\begin{array}{l}\text { Compares GA } \\
\text { as Ist line and } \\
\text { switch to GA }\end{array}$ \\
\hline $\begin{array}{l}\text { Gerber, } \\
2017^{59}\end{array}$ & Canada & $\mathrm{AH}, \mathrm{DC}$ & 12 months & $N=2,781$ & $\begin{array}{l}\text { Age: <35 (25\%), } \\
35-55(61.7 \%), 55-65 \\
(11.8 \%),>65(1.5 \%) ; \\
\text { Gender: 73.8\% Female; } \\
\text { Subtype: NR; MS dura- } \\
\text { tion: NR; EDSS: NR }\end{array}$ & $\begin{array}{l}\text { Retrospective/ } \\
\text { administrative } \\
\text { data }\end{array}$ & $\begin{array}{l}\text { IFN } \beta \text {-1a, } \\
\text { IFN } \beta \text {-1b, GA, } \\
\text { FTY, TFN, DMF }\end{array}$ & \\
\hline
\end{tabular}




\section{TABLE 1 Characteristics of Included Objective Studies (continued)}

\begin{tabular}{|c|c|c|c|c|c|c|c|c|}
\hline $\begin{array}{l}\text { Author, } \\
\text { year }\end{array}$ & Country & $\begin{array}{l}\text { Study } \\
\text { type }\end{array}$ & $\begin{array}{c}\text { Observation } \\
\text { length }\end{array}$ & Sample size & $\begin{array}{l}\text { Age, gender, subtype, } \\
\text { MS duration, EDSS }\end{array}$ & Design/setting & DMT(s) & Comments \\
\hline $\begin{array}{l}\text { Hansen, } \\
2015^{10}\end{array}$ & Germany & $\mathrm{AH}$ & 24 months & $N=50,057$ & $\begin{array}{l}\text { Age: NR; Gender: } \\
\text { NR; Subtype: NR; MS } \\
\text { duration: NR; EDSS: NR }\end{array}$ & $\begin{array}{l}\text { Retrospective/ } \\
\text { pharmacy } \\
\text { claims } \\
\text { database }\end{array}$ & $\begin{array}{l}\text { IFN } \beta-1 a \text {, } \\
\text { IFN } \beta-1 b, G A\end{array}$ & $\begin{array}{l}\text { Demographics } \\
\text { not reported }\end{array}$ \\
\hline $\begin{array}{l}\text { Johnson, } \\
2017^{12}\end{array}$ & USA & $\mathrm{AH}$ & 12 months & $N=1,498$ & $\begin{array}{l}\text { Age: } M=48.1, S D=10.7 \\
\text { years; Gender: } 76.7 \% \\
\text { Female; Subtype: NR; } \\
\text { MS duration: NR; EDSS: } \\
\text { NR }\end{array}$ & $\begin{array}{l}\text { Retrospective/ } \\
\text { database } \\
\text { claims }\end{array}$ & FTY, DMF, TFN & \\
\hline $\begin{array}{l}\text { Jones, } \\
2013^{60}\end{array}$ & USA & $\mathrm{AH}$ & 24 months & $N=5,825$ & $\begin{array}{l}\text { Age: } M=44.4, S D=10.1 \\
\text { years; Gender: NR; } \\
\text { Subtype: RRMS; MS } \\
\text { duration: NR; EDSS: NR }\end{array}$ & $\begin{array}{l}\text { Retrospective/ } \\
\text { shared solution } \\
\text { program }\end{array}$ & $\mathrm{GA}$ & $\begin{array}{l}\text { Participants } \\
\text { were enrolled } \\
\text { in a medication } \\
\text { support } \\
\text { program }\end{array}$ \\
\hline $\begin{array}{l}\text { Kleinman, } \\
2010^{61}\end{array}$ & USA & $\mathrm{AH}$ & 12 months & $N=358$ & $\begin{array}{l}\text { Age: } M=40.7, S D=5.9 \\
\text { years; Gender: } 62.7 \% \\
\text { Female; Subtype: NR; } \\
\text { MS duration: NR; EDSS: } \\
\text { NR }\end{array}$ & $\begin{array}{l}\text { Retrospective/ } \\
\text { claims } \\
\text { database }\end{array}$ & $\begin{array}{l}\text { IFN } \beta-1 a \text {, } \\
\text { IFN } \beta-1 b, G A\end{array}$ & $\begin{array}{l}\text { Participants } \\
\text { had to be } \\
\text { employed for } \\
\text { this study }\end{array}$ \\
\hline $\begin{array}{l}\text { Kleiter, } \\
2017^{62}\end{array}$ & Germany & $\mathrm{AH}$ & 24 weeks & $\begin{array}{c}\mathrm{N}=151 \\
\text { (enrolled); } \\
\mathrm{N}=143 \\
\text { (analyzed) }\end{array}$ & $\begin{array}{l}\text { Age: } M=41.2, S D=11.5 \\
\text { years; Gender: } 69.2 \% \\
\text { Female; Subtype: } \\
\text { RRMS, CIS; MS dura- } \\
\text { tion: }(n=105), M=15.2, \\
\text { SD }=29.4 \text { months; } \\
\text { EDSS: }(n=105) \text { median, } \\
2.0 \text { (range, } 0-6.5)\end{array}$ & $\begin{array}{l}\text { Prospective/ } \\
\text { multicentre }\end{array}$ & IFN $\beta-1$ - s.c. & \\
\hline $\begin{array}{l}\text { Kozma, } \\
2014^{63}\end{array}$ & USA & $\mathrm{AH}$ & 24 months & $N=4,606$ & $\begin{array}{l}\text { Age: } M=46.0, S D=9.4 \\
\text { years; Gender: } 78.7 \% \\
\text { Female; Subtype: NR; } \\
\text { MS duration: NR; EDSS: } \\
\text { NR }\end{array}$ & $\begin{array}{l}\text { Retrospective/ } \\
\text { database } \\
\text { claims }\end{array}$ & $\begin{array}{l}\text { IFN } \beta-1 a \text {, } \\
\text { IFN } \beta-1 b, G A\end{array}$ & \\
\hline $\begin{array}{l}\text { Krol, } \\
2017^{64}\end{array}$ & $\begin{array}{c}\text { Netherlands } \\
\text { and Germany }\end{array}$ & $\mathrm{AH}$ & Up to 5 years & $N=1,682$ & $\begin{array}{l}\text { Age: } M=40.1, S D=10.7 \\
\text { years; Gender: } 72.5 \% \\
\text { Female; Subtype: NR; } \\
\text { MS duration: NR; EDSS: } \\
\text { NR }\end{array}$ & $\begin{array}{l}\text { Retrospective/ } \\
\text { multinational }\end{array}$ & IFN $\beta$-1a s.c. & $\begin{array}{l}\text { No demo- } \\
\text { graphic data } \\
\text { reported } \\
\text { for the } \\
\text { Netherlands }\end{array}$ \\
\hline $\begin{array}{l}\text { Lafata, } \\
2008^{19}\end{array}$ & USA & $\mathrm{AH}$ & 24 months & $N=224$ & $\begin{array}{l}\text { Age: } M=47.6, S D=10.4 \\
\text { years; Gender: } 77.2 \% \\
\text { Female; Subtype: } \\
\text { RRMS; MS duration: } \\
\text { NR; EDSS: NR }\end{array}$ & $\begin{array}{l}\text { Retrospective/ } \\
\text { multispecialty } \\
\text { practice }\end{array}$ & $\begin{array}{l}\text { GA, IFN } \beta-1 a \text {, } \\
\text { IFN } \beta-1 b\end{array}$ & $\begin{array}{l}\text { Insured } \\
\text { patients }\end{array}$ \\
\hline $\begin{array}{l}\text { Lebrun- } \\
\text { Frenay, } \\
2019^{65}\end{array}$ & France & DC & Up to 5 years & $\begin{array}{c}\mathrm{N}=881 \\
\text { (enrolled); } \\
\mathrm{N}=852 \\
\text { (analyzed) }\end{array}$ & $\begin{array}{l}\text { Age: } M=39.9, S D=10.6 \\
\text { years; Gender: } 75.8 \% \\
\text { Female; Subtype: } \\
\text { RRMS; MS duration: } \\
M=8.1, S D=7.5 \text { years; } \\
\text { EDSS: } M=2.3, S D=1.7 \\
(n=716)\end{array}$ & $\begin{array}{l}\text { Prospective/ } \\
\text { database } \\
\text { claims }\end{array}$ & $\mathrm{GA}$ & \\
\hline
\end{tabular}




\section{TABLE 1 Characteristics of Included Objective Studies (continued)}

\begin{tabular}{|c|c|c|c|c|c|c|c|c|}
\hline $\begin{array}{l}\text { Author, } \\
\text { year }\end{array}$ & Country & $\begin{array}{l}\text { Study } \\
\text { type }\end{array}$ & $\begin{array}{c}\text { Observation } \\
\text { length }\end{array}$ & Sample size & $\begin{array}{l}\text { Age, gender, subtype, } \\
\text { MS duration, EDSS }\end{array}$ & Design/setting & DMT(s) & Comments \\
\hline $\begin{array}{l}\text { Lugaresi, } \\
2012^{66}\end{array}$ & Italy & $\mathrm{AH}$ & 12 weeks & $\begin{array}{c}\mathrm{N}=120 \\
\text { (recruited); } \\
\mathrm{N}=119 \text { (intent- } \\
\text { to-treat } \\
\text { population); } \\
\mathrm{N}=109 \\
\text { (completed } \\
\text { study) }\end{array}$ & $\begin{array}{l}\text { Age: } M=37.9, S D=9.7 \\
\text { years; Gender: } 75.6 \% \\
\text { Female; Subtype: } \\
\text { RRMS; MS duration: } \\
M=5.8, S D=5.3 \text { years; } \\
\text { EDSS: } M=2.1, S D=1.2\end{array}$ & $\begin{array}{l}\text { Prospective/ } \\
\text { multicentre }\end{array}$ & IFN $\beta-1$ a s.c. & \\
\hline $\begin{array}{l}\text { McKay, } \\
2017^{67}\end{array}$ & Canada & $\mathrm{AH}$ & 24 months & $\begin{array}{c}\mathrm{N}=326 \\
\text { (included); } \\
\mathrm{N}=135 \text { (DMT } \\
\text { users) }\end{array}$ & $\begin{array}{l}\text { Age: } M=48.7, S D=11.9 \\
\text { years; Gender: } 74.5 \% \\
\text { Female; Subtype: } \\
\text { RRMS, SPMS, PPMS; } \\
\text { MS duration: } M=15.3 \text {, } \\
\text { SD = 9.9 years; EDSS: } \\
\text { Median, } 2.5 \text { (IQR, 2.0- } \\
\text { 4.0) }\end{array}$ & $\begin{array}{l}\text { Retrospective/ } \\
\text { pharmacy } \\
\text { claims }\end{array}$ & $\begin{array}{l}\text { IFN } \beta-1 a \text {, } \\
\text { IFN } \beta-1 b, G A\end{array}$ & \\
\hline $\begin{array}{l}\text { Melesse, } \\
2017^{35}\end{array}$ & Canada & $\mathrm{AH}, \mathrm{DC}$ & $M=7.8$ years & $N=721$ & $\begin{array}{l}\text { Age: } M=37.6, S D=9.8 \\
\text { years; Gender: } 74.2 \% \\
\text { Female; Subtype: NR; } \\
\text { MS duration: NR; EDSS: } \\
\text { NR }\end{array}$ & $\begin{array}{l}\text { Retrospective/ } \\
\text { administrative } \\
\text { data }\end{array}$ & $\begin{array}{l}\text { IFN } \beta-1 a \text {, } \\
\text { IFN } \beta-1 b, G A\end{array}$ & $\begin{array}{l}\text { Subset study } \\
\text { of Evans, 2016; } \\
\text { 2017, specific } \\
\text { to first DMT } \\
\text { prescribed }\end{array}$ \\
\hline $\begin{array}{l}\text { Moccia, } \\
2016^{68}\end{array}$ & Italy & DC & $\begin{array}{c}>5 \text { years; } \\
M=7.9, S D=3.8 \\
\text { years }\end{array}$ & $N=499$ & $\begin{array}{l}\text { Age: } M=32.3, S D=8.2 \\
\text { years; Gender: } 63.1 \% \\
\text { Female; Subtype: } \\
\text { RRMS; } M S \text { duration; } \\
M=2.7, S D=2.7 \text { years; } \\
\text { EDSS: } M=2.0, S D=0.6\end{array}$ & $\begin{array}{l}\text { Retrospective/ } \\
\text { clinical data }\end{array}$ & $\begin{array}{l}\text { IFN } \beta-1 a \\
\text { IFN } \beta-1 b\end{array}$ & $\begin{array}{l}\text { Newly } \\
\text { commenced } \\
\text { on IFN } \beta \text {-1a \& } \\
\text { 1b as first DMT }\end{array}$ \\
\hline $\begin{array}{l}\text { Moccia, } \\
2015^{69}\end{array}$ & Italy & $\mathrm{AH}$ & Up to 4 years & $N=114$ & $\begin{array}{l}\text { Age: } M=35.8, S D=10.4 \\
\text { years; Gender: } 71.1 \% \\
\text { Female; Subtype: } \\
\text { RRMS; } M S \text { duration: } \\
M=8.1, S D=9.1 \text { years; } \\
\text { EDSS: } M=2.8, S D=0.8\end{array}$ & $\begin{array}{l}\text { Retrospective/ } \\
\text { clinical data }\end{array}$ & IFN $\beta$-1a s.c & \\
\hline $\begin{array}{l}\text { Munsell, } \\
2017^{70}\end{array}$ & USA & $\mathrm{AH}, \mathrm{DC}$ & 12 months & $\mathrm{N}=8,382$ & $\begin{array}{l}\text { Age: } M=43.1, S D=N R \\
\text { years; Gender: } 76.7 \% \\
\text { Female; Subtype: NR; } \\
\text { MS duration: NR; EDSS: } \\
\text { NR }\end{array}$ & $\begin{array}{l}\text { Retrospective/ } \\
\text { claims } \\
\text { database }\end{array}$ & $\begin{array}{l}\text { IFN } \beta \text {-1a, } \\
\text { IFN } \beta-1 b, \text { GA, } \\
\text { TFN, FTY, DMF }\end{array}$ & $\begin{array}{l}\text { Newly initiated } \\
\text { treatment }\end{array}$ \\
\hline $\begin{array}{l}\text { Oleen- } \\
\text { Burkey, } \\
2011^{71}\end{array}$ & USA & $\mathrm{AH}$ & 24 months & $N=839$ & $\begin{array}{l}\text { Age: } M=45.2, S D=10.4 \\
\text { years; Gender: } 81.3 \% \\
\text { Female; Subtype: NR; } \\
\text { MS duration: NR; EDSS: } \\
\text { NR }\end{array}$ & $\begin{array}{l}\text { Retrospective/ } \\
\text { administrative } \\
\text { claims data }\end{array}$ & GA & \\
\hline $\begin{array}{l}\text { Paolicelli, } \\
2016^{72}\end{array}$ & Italy & $\mathrm{AH}$ & 24 months & $\begin{array}{c}N=384 \\
\text { (recruited); } \\
N=343 \\
(12 \text { months); } \\
N=150 \\
\text { (24 months) }\end{array}$ & $\begin{array}{l}\text { Age: } M=36.0, S D=9.2 \\
\text { years; Gender: } 67.2 \% \\
\text { Female; Subtype: } \\
\text { RRMS; MS duration: } \\
\text { Median, } 74 \text { months } \\
\text { (range, } 0-398) ; \text { EDSS: } \\
\text { Median, } 2 \text { months } \\
\text { (range, } 0-6 \text { ) }\end{array}$ & $\begin{array}{l}\text { Prospective/ } \\
\text { clinical } \\
\text { database }\end{array}$ & IFN $\beta$-1a s.c. & \\
\hline
\end{tabular}




\section{TABLE 1 Characteristics of Included Objective Studies (continued)}

\begin{tabular}{|c|c|c|c|c|c|c|c|c|}
\hline $\begin{array}{l}\text { Author, } \\
\text { year }\end{array}$ & Country & $\begin{array}{l}\text { Study } \\
\text { type }\end{array}$ & $\begin{array}{c}\text { Observation } \\
\text { length }\end{array}$ & Sample size & $\begin{array}{l}\text { Age, gender, subtype, } \\
\text { MS duration, EDSS }\end{array}$ & Design/setting & DMT(s) & Comments \\
\hline $\begin{array}{l}\text { Pedersen, } \\
2018^{73}\end{array}$ & Demark & $\mathrm{AH}$ & 12 weeks & $\begin{array}{c}\mathrm{N}=60 \\
\text { (recruited); } \\
\mathrm{N}=54 \\
\text { (analyzed) }\end{array}$ & $\begin{array}{l}\text { Age: } M=43.7, S D=7.9 \\
\text { years; Gender: } 83.3 \% \\
\text { Female; Subtype: } \\
\text { RRMS; MS duration: } \\
\text { M=6.7, SD = 4.5 years; } \\
\text { EDSS: Median, } 2.75 \\
\text { (range, } 0-5 \text { ) }\end{array}$ & $\begin{array}{l}\text { Prospective/ } \\
\text { multicentre }\end{array}$ & IFN $\beta$-1a s.c. & \\
\hline $\begin{array}{l}\text { Prosperini, } \\
2019^{74}\end{array}$ & Italy & DC & 12 months & $\begin{array}{l}\text { Prematching: } \\
\mathrm{N}=621 ; \mathrm{PEG}, \\
\mathrm{N}=196, \mathrm{DMF} \\
\mathrm{N}=265, \mathrm{TFN} \\
\mathrm{N}=160\end{array}$ & $\begin{array}{l}\text { Age: } M=41.9, S D=10.7 \\
\text { years; Gender: } 69.3 \% \\
\text { Female; Subtype: } \\
\text { RRMS; MS duration: } \\
\text { M=11.0, SD = } 7.4 \text { years; } \\
\text { EDSS: PEG (1.0 [0-5.5]); } \\
\text { DMF (1.5 [0-0.5]); TFN } \\
(2.5 \text { [0-5.5]) }\end{array}$ & $\begin{array}{l}\text { Retrospective/ } \\
\text { multicentre }\end{array}$ & TFN, DMF, PEG & $\begin{array}{l}\text { Data } \\
\text { propensity } \\
\text { matched; } \\
\text { Prematching } \\
\text { cohort } \\
\text { demographics } \\
\text { displayed }\end{array}$ \\
\hline $\begin{array}{l}\text { Sabido- } \\
\text { Espin, } \\
2017^{75}\end{array}$ & USA & $\mathrm{AH}, \mathrm{DC}$ & 36 months & $N=5,956$ & $\begin{array}{l}\text { Age: } M=44.0, S D=10.7 \\
\text { years; Gender: } 74.7 \% \\
\text { Female; Subtype: NR; } \\
\text { MS duration; NR; EDSS: } \\
\text { NR }\end{array}$ & $\begin{array}{l}\text { Retrospective/ } \\
\text { insurance } \\
\text { claims } \\
\text { database }\end{array}$ & IFN $\beta$-1a s.c & \\
\hline $\begin{array}{l}\text { Sanchirico, } \\
2019^{76}\end{array}$ & USA & $\mathrm{AH}$ & 12 months & $N=7,072$ & $\begin{array}{l}\text { Age: } M=55.0, S D=10.0 \\
\text { years; Gender: } 78.3 \% \\
\text { Female; Subtype: NR; } \\
\text { MS duration: NR; EDSS: } \\
\text { NR }\end{array}$ & $\begin{array}{l}\text { Retrospective/ } \\
\text { medical and } \\
\text { pharmacy } \\
\text { claims }\end{array}$ & $\begin{array}{l}\text { GA, IFN } \beta-1 a \\
\text { IFN } \beta-1 b, T F N \text {, } \\
\text { DMF, FTY }\end{array}$ & \\
\hline $\begin{array}{l}\text { Schreiber, } \\
2018^{77}\end{array}$ & Denmark & $\mathrm{AH}$ & 12 months & $\begin{array}{c}\mathrm{N}=195 \\
\text { (enrolled); } \\
\mathrm{N}=126 \\
\text { (12 months) }\end{array}$ & $\begin{array}{l}\text { Age: } M=41.0, S D=8.7 \\
\text { years; Gender: } 66.7 \% \\
\text { Female; Subtype: } \\
\text { RRMS; } M S \text { duration: } \\
M=9.0, S D=6.0 \text { years; } \\
\text { EDSS: } M=3.0, S D=1.7\end{array}$ & $\begin{array}{l}\text { Prospective/ } \\
\text { multicentre }\end{array}$ & FTY & $\begin{array}{l}\text { Only Includes } \\
\text { intervention } \\
\text { study, but } \\
\text { observational } \\
\text { data used }\end{array}$ \\
\hline $\begin{array}{l}\text { Settle, } \\
2016^{30}\end{array}$ & USA & $\mathrm{AH}$ & 6 months & $\begin{array}{c}\mathrm{N}=30 \\
\text { (recruited); } \\
\mathrm{N}=13 \\
\text { (routine care); } \\
\mathrm{N}=17 \\
\text { (intervention) }\end{array}$ & $\begin{array}{l}\text { Age: } M=48.0, S D=10.8 \\
\text { years; Gender: } 50.0 \% \\
\text { Female; Subtype: } \\
\text { RRMS, SPMS; MS dura- } \\
\text { tion: NR; EDSS: Median } \\
\text { 3-3.5, range 2.0-8.0 }\end{array}$ & $\begin{array}{l}\text { Prospective/ } \\
\text { veterans' affair } \\
\text { medical centre }\end{array}$ & IFN $\beta-1$ a i.m. & $\begin{array}{l}\text { Compares } \\
\text { usual care to } \\
\text { online web- } \\
\text { based tool; } \\
\text { Veterans' Affair } \\
\text { Medical Centre } \\
\text { sample }\end{array}$ \\
\hline $\begin{array}{l}\text { Shao, } \\
2018^{78}\end{array}$ & USA & $\mathrm{AH}$ & $\mathrm{M}=281.56$ days & $N=6,003$ & $\begin{array}{l}\text { Age: } M=46.0, S D=10.1 \\
\text { years; Gender: } 77.5 \% \\
\text { Female; Subtype: NR; } \\
\text { MS duration: NR; EDSS: } \\
\text { NR }\end{array}$ & $\begin{array}{l}\text { Retrospective/ } \\
\text { commercial } \\
\text { claims } \\
\text { database }\end{array}$ & $\begin{array}{l}\text { IFN } \beta-1 a \text {, } \\
\text { IFN } \beta-1 b, G A\end{array}$ & \\
\hline $\begin{array}{l}\text { Smith, } \\
2015^{79}\end{array}$ & USA & DC & $M=554.2$ days & $N=8,107$ & $\begin{array}{l}\text { Age: } M=43.1, S D=10.7 \\
\text { years; Gender: } 76.2 \% \\
\text { Female; Subtype: NR; } \\
\text { MS duration: NR; EDSS: } \\
\text { NR }\end{array}$ & $\begin{array}{l}\text { Retrospective/ } \\
\text { insurance } \\
\text { claims } \\
\text { database }\end{array}$ & IFN $\beta-1 a$ & $\begin{array}{l}\text { Newly } \\
\text { commenced } \\
\text { IFN } \beta-1 a\end{array}$ \\
\hline $\begin{array}{l}\text { Solsona, } \\
2017^{80}\end{array}$ & Spain & $\mathrm{AH}$ & $\begin{array}{c}\text { Median, } 979 \\
\text { days }\end{array}$ & $\mathrm{N}=110$ & $\begin{array}{l}\text { Age: } M=38.8, S D=9.3 \\
\text { years; Gender: } 66.4 \% \\
\text { Female; Subtype: NR; } \\
\text { MS duration: NR; EDSS: } \\
\text { Median, } 1.5 \text { (range, } \\
0-4.5 \text { ) }\end{array}$ & $\begin{array}{l}\text { Retrospective/ } \\
\text { multiple } \\
\text { sclerosis } \\
\text { clinic data }\end{array}$ & IFN $\beta$-1a s.c. & \\
\hline
\end{tabular}




\section{TABLE 1 Characteristics of Included Objective Studies (continued)}

\begin{tabular}{|c|c|c|c|c|c|c|c|c|}
\hline $\begin{array}{l}\text { Author, } \\
\text { year }\end{array}$ & Country & $\begin{array}{l}\text { Study } \\
\text { type }\end{array}$ & $\begin{array}{c}\text { Observation } \\
\text { length }\end{array}$ & Sample size & $\begin{array}{l}\text { Age, gender, subtype, } \\
\text { MS duration, EDSS }\end{array}$ & Design/setting & DMT(s) & Comments \\
\hline $\begin{array}{l}\text { Steinberg, } \\
2010^{36}\end{array}$ & USA & $\mathrm{AH}$ & $\begin{array}{l}3 \text { years; } \\
\text { reported in } \\
\text { 12-month } \\
\text { periods }\end{array}$ & $N=1,606$ & $\begin{array}{l}\text { Age: } M=51.8, S D=9.8 \\
\text { years; Gender: } 76.0 \% \\
\text { Female; Subtype: } \\
\text { RRMS; MS duration: } \\
\text { NR; EDSS: NR }\end{array}$ & $\begin{array}{l}\text { Retrospective/ } \\
\text { database } \\
\text { claims }\end{array}$ & $\begin{array}{l}\text { IFN } \beta-1 a \text {, } \\
\text { IFN } \beta-1 b\end{array}$ & \\
\hline $\begin{array}{l}\text { Stockl, } \\
2010^{81}\end{array}$ & USA & $\mathrm{AH}, \mathrm{DC}$ & 8 months & $N=468$ & $\begin{array}{l}\text { Age: } M=53.2, S D=10.2 \\
\text { years; Gender: } 83.3 \% \\
\text { Female; Subtype: NR; } \\
\text { MS duration; NR; EDSS: } \\
\text { NR }\end{array}$ & $\begin{array}{l}\text { Retrospective/ } \\
\text { insurance } \\
\text { database } \\
\text { claims }\end{array}$ & $\begin{array}{l}\text { IFN } \beta-1 a \text {, } \\
\text { IFN } \beta-1 b, G A\end{array}$ & $\begin{array}{l}\text { Disease } \\
\text { management } \\
\text { program } \\
\text { compared } \\
\text { to retail and } \\
\text { specialty } \\
\text { pharmacy } \\
\text { participant }\end{array}$ \\
\hline $\begin{array}{l}\text { Tan, } \\
2011^{82}\end{array}$ & USA & $\mathrm{AH}$ & 12 months & $N=2,446$ & $\begin{array}{l}\text { Age: } M=41.9, S D=10.1 \\
\text { years; Gender: } 74.9 \% \\
\text { Female; Subtype: NR; } \\
\text { MS duration: NR; EDSS: } \\
\text { NR }\end{array}$ & $\begin{array}{l}\text { Retrospective/ } \\
\text { insurance } \\
\text { database } \\
\text { claims }\end{array}$ & $\begin{array}{l}\text { IFN } \beta-1 a \text {, } \\
\text { IFN } \beta-1 b, G A\end{array}$ & \\
\hline $\begin{array}{l}\text { Vieira, } \\
2019^{83}\end{array}$ & USA & $\mathrm{AH}$ & 24 months & $N=4,563$ & $\begin{array}{l}\text { Age: } M=46.7, S D=9.9 \\
\text { years; Gender: } 78.2 \% \\
\text { Female; Subtype: NR; } \\
\text { MS duration: NR; EDSS: } \\
\text { NR }\end{array}$ & $\begin{array}{l}\text { Retrospective/ } \\
\text { database }\end{array}$ & FTY, TFN & \\
\hline $\begin{array}{l}\text { Warrender- } \\
\text { Sparkes, } \\
2016^{84}\end{array}$ & International & DC & 12 months & $N=2,640$ & $\begin{array}{l}\text { Age: } M=32.9, S D=10.0 \\
\text { years; Gender: } 70.3 \% \\
\text { Female; Subtype: } \\
\text { RRMS, SPMS, CIS; MS } \\
\text { duration: NR; EDSS: } \\
\text { Median, } 2 \text { (IQR 1, 2.5) }\end{array}$ & $\begin{array}{l}\text { Retrospective/ } \\
\text { MBase registry }\end{array}$ & $\begin{array}{l}\text { IFN } \beta-1 a \text {, } \\
\text { IFN } \beta-1 b, G A \text {, } \\
\text { FTY }\end{array}$ & $\begin{array}{l}\text { Newly } \\
\text { diagnosed, } \\
\text { commencing } \\
\text { DMT }\end{array}$ \\
\hline $\begin{array}{l}\text { Williams, } \\
2018^{85}\end{array}$ & USA & $\mathrm{AH}$ & 12 months & $N=381$ & $\begin{array}{l}\text { Age: } M=41.8, S D=10.9 \\
\text { years; Gender: } 82.8 \% \\
\text { Female; Subtype: NR; } \\
\text { MS duration: NR; EDSS: } \\
\text { NR }\end{array}$ & $\begin{array}{l}\text { Retrospective/ } \\
\text { administrative } \\
\text { database } \\
\text { claims }\end{array}$ & FTY, GA & $\begin{array}{l}\text { Hispanic } \\
\text { and African } \\
\text { American sub- } \\
\text { groups }\end{array}$ \\
\hline $\begin{array}{l}\text { Zecca, } \\
2017^{38}\end{array}$ & Switzerland & $\mathrm{AH}$ & 9 months & $\begin{array}{c}\mathrm{N}=56 \\
\text { (observation); } \\
\mathrm{N}=53 \text { ( } \\
\text { evaluable data) }\end{array}$ & $\begin{array}{l}\text { Age: Median, } 49.0 \text { (IQR } \\
\text { 38, 55); Gender: } 77.4 \% \\
\text { Female; Subtype: } \\
\text { RMMS; MS duration: } \\
\text { Median, } 6.5 \text { years (IQR, } \\
\text { 38, 55); EDSS: Median, } \\
2 \text { (IQR 1,3.3) }\end{array}$ & $\begin{array}{l}\text { Retrospective } \\
\text { and } \\
\text { prospective/ } \\
\text { multicentre }\end{array}$ & IFN $\beta$-1a s.c & $\begin{array}{l}\text { Includes a } \\
\text { retrospective } \\
\text { and } \\
\text { prospective } \\
\text { observation } \\
\text { period }\end{array}$ \\
\hline
\end{tabular}

continued on next page

travel compensation $(\mathrm{N}=31 ; 50.8 \%)$, or they were employed by a pharmaceutical company $(\mathrm{N}=28 ; 45.9 \%)$. While unsurprising, given pharmaceutical companies interest in understanding real-world adherence figures for their products, this interest warrants remark.

\section{ADHERENCE RATES}

Medication adherence was most often measured using the MPR $(\mathrm{N}=23)$, followed by the PDC $(\mathrm{N}=13)$, and electronic autoinjectors $(\mathrm{N}=12)$. In addition, medication adherence was also measured with pill count $(\mathrm{PC} ; \mathrm{N}=3)$, MEMS $(\mathrm{N}=2)$, and injection count (IC; $\mathrm{N}=1)$.

Binary Adherence $(\geq 80 \%)$. Using a binary measure, 32 studies reported injectable adherence and 10 reported oral adherence (see Supplementary Table 1 and 2). For studies with a less than 8-month observation period, autoinjector adherence for injectable DMTs $(\mathrm{N}=4)$ ranged between 80.5\% and 93.0\%, while one study using MEMS reported 


\section{TABLE 1 Characteristics of Included Objective Studies (continued)}

\begin{tabular}{|c|c|c|c|c|c|c|c|c|}
\hline $\begin{array}{l}\text { Author, } \\
\text { year }\end{array}$ & Country & $\begin{array}{l}\text { Study } \\
\text { type }\end{array}$ & $\begin{array}{c}\text { Observation } \\
\text { length }\end{array}$ & Sample size & $\begin{array}{l}\text { Age, gender, subtype, } \\
\text { MS duration, EDSS }\end{array}$ & Design/setting & DMT(s) & Comments \\
\hline $\begin{array}{l}\text { Zhang, } \\
2017^{86}\end{array}$ & Canada & $\mathrm{AH}$ & 12 months & $N=801$ & $\begin{array}{l}\text { Age: } M=41.5, S D=9.5 \\
\text { years; Gender: } 76.0 \% \\
\text { Female; Subtype: NR; } \\
\text { MS duration: NR; EDSS: } \\
\text { Median, } 3 \text { (IQR 2,4) }\end{array}$ & $\begin{array}{l}\text { Prospective/ } \\
\text { clinical and } \\
\text { administrative } \\
\text { database }\end{array}$ & $\begin{array}{l}\text { IFN } \beta-1 a \text {, } \\
\text { IFN } \beta-1 b, G A\end{array}$ & \\
\hline $\begin{array}{l}\text { Zhornitsky, } \\
2015^{87}\end{array}$ & Canada & DC & 18 years & $N=1,471$ & $\begin{array}{l}\text { Age: } M=38.4, S D=9.3 \\
\text { years; Gender: } 75.3 \% \\
\text { Female; Subtype: } \\
\text { RRMS; MS duration: } \\
\text { M=6.0, SD=6.7 years; } \\
\text { EDSS: Median, 2.0 } \\
\text { years (IQR: } 1.5-3.0 ; \\
\text { Range, } 0-8.0 \text { ) }\end{array}$ & $\begin{array}{l}\text { Prospective/ } \\
\text { MS clinic }\end{array}$ & $\begin{array}{l}\text { IFN } \beta-1 a \text {, } \\
\text { IFN } \beta-1 b, G A\end{array}$ & \\
\hline $\begin{array}{l}\text { Zimmer, } \\
2017^{88}\end{array}$ & Switzerland & $\mathrm{AH}, \mathrm{DC}$ & 24 months & $N=98$ & $\begin{array}{l}\text { Age: Median, } 41.0 \\
\text { years (IQR: 31.0, 46.0; } \\
\text { Range, 22.0-71.0); } \\
\text { Gender: 79.6\% Female; } \\
\text { Subtype: RRMS; MS } \\
\text { duration: Median, } 4.6 \\
\text { years (IQR: 1.0, 11.4; } \\
\text { Range, 0.2-36.4); EDSS: } \\
\text { NR }\end{array}$ & $\begin{array}{l}\text { Prospective/ } \\
\text { MS clinic data- } \\
\text { base }\end{array}$ & FTY & \\
\hline
\end{tabular}

Notes: Unless otherwise stated, IFN $\beta$-1a includes both intramuscular and subcutaneous administration. All studies are observational unless otherwise stated. Sample sizes included are reported statistics, not total sample, unless specified. Only baseline and preintervention data are reported for intervention studies. Where required, means and standard deviations were combined excluding IV medications data, using sample group statistics through an online calculator: https:// www.statstodo.com/CombineMeansSDs_Pgm.php.

$A H=$ adherence; $C I S=$ clinically isolated syndrome; $D C=$ discontinuation; $D M F=$ dimethyl fumarate; $E D S S=$ expanded disability status scale; $F T Y=$ fingolimod; $\mathrm{GA}=$ glatiramer acetate; IFN $\beta$-1 $a=$ interferon beta-1a; IFN $\beta$-Ia i.m = interferon beta-1a intramuscular; IFN $\beta$-Ia s. $c=$ interferon beta-1a subcutaneous; IFN $\beta$ $1 b=$ interferon bet $a-1 b ; I Q R=$ interquartile range; $M P R=$ medication possession ratio; $N R=$ not reported; $p w R R M S=$ people with relapse remitting multiple sclerosis; $P P M S=$ primary progressive multiple sclerosis; $P D C=$ proportion of days covered; $P E G=$ pegylated interferon; $P M=$ propensity matched; RRMS= relapse remitting multiple sclerosis; SPMS = secondary progressive multiple sclerosis; TFN=teriflunomide.

adherence of $82.0 \%$, and another study reported MPR adherence of $53.0 \%$. There was only one comparative binary oral DMT study with a MPR adherence of $74.0 \%$.

For 12-month observation studies, MPR adherence for injectable DMTs $(\mathrm{N}=7)$ and PDC $(\mathrm{N}=6)$ ranged, respectively, between $47.0 \%$ and $77.4 \%$ and $46.0 \%$ to $90.0 \%$. Whereas MPR adherence for oral DMTs $(\mathrm{N}=7)$ ranged between $53.0 \%$ and $89.2 \%$. PDC adherence $(\mathrm{N}=6)$ ranged between $60.4 \%$ and $94.0 \%$. Meta-analysis for at least $80 \%$ oral and injectable DMT adherence did not find a statistical difference between pooled estimates for MPR (injectable: 62.0\%; 95\% CI: 51.0\%-73.0\%; oral: 79.0\%; 95\% CI: 61.0\%-95.0\%) or PDC (injectable: $80.0 \%$; 95\% CI: 66.0\%-92.0\%; oral: $80.0 \%$; 95\% CI: 52.0\%-100.0\%) measures (Supplementary Figure 1, available in online article).

For studies conducted over 24 months, mean MPR adherence for injectable DMTs $(\mathrm{N}=7)$ ranged between $35.0 \%$ to $79.0 \%$, and the results from two studies reporting mean PDC were $67.3 \%$ and $74.7 \%$. There were two oral DMT adherence studies for 24 months. One study reported an MPR of $64.7 \%$, and the second study classified adherence into four categories using mean PDC, from perfect adherence (100\%) to nonadherence $(<85.8 \%)$. No participants were classified as nonadherent, possibly due to methodological error, given that $22.4 \%$ of participants who discontinued treatment during the observation period were excluded from analysis.

Of the seven 36-month binary adherence studies for injectable DMTs, one reported an MPR of 51.7\%. PDC adherence $(\mathrm{N}=2)$ was reported as $42.4 \%$ and $86.0 \%$, and autoinjector adherence studies $(\mathrm{N}=4)$ ranged between $82.9 \%$ and $95.6 \%$. There were no 36-month binary oral adherence studies.

Continuous Adherence. Twenty-nine studies reported mean adherence on a continuous scale: 21 injectable and eight oral DMTs. Two studies reported a less than eightmonth continuous adherence for injectable DMTs. One study measured autoinjector adherence. The other study combined injection count and MPR data with a mean 


\section{FIGURE 2 Meta-Analysis of Mean Adherence Rates}

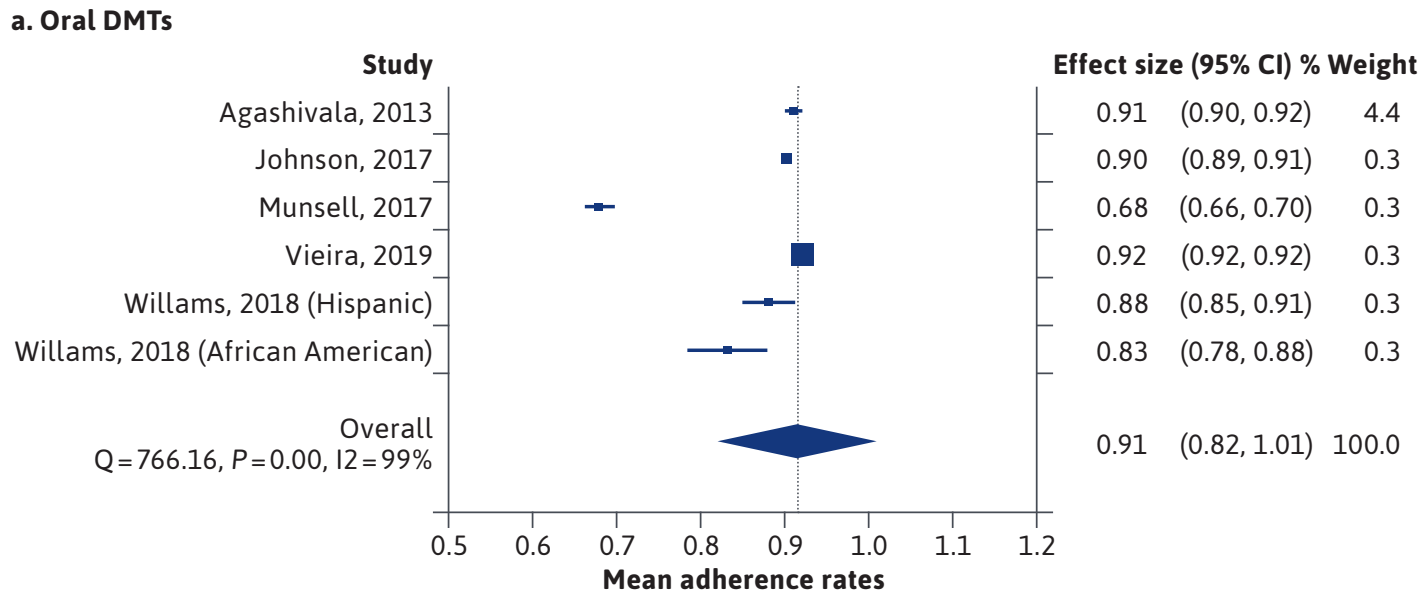

\section{b. Injectable DMTs}

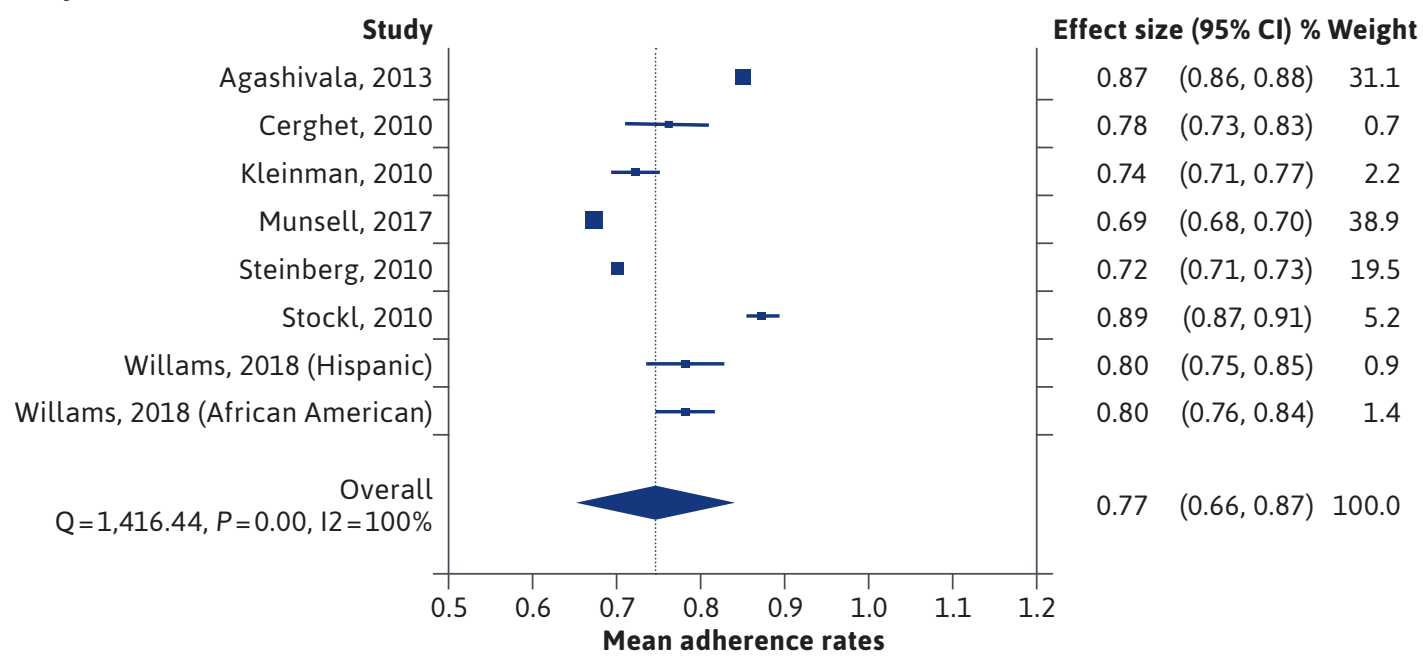

Notes: Pooled subgroup results are presented (overall pooled estimates not available). Weights are from Doi's IVHet Model. Effect=mean adherence ratios.

adherence, respectively, of $89.0 \%$ and $95.0 \%$. One small study (25 participants) reported oral DMT adherence using PC (91.0\%) and compared it to MEMS cap results (90.0\%).

Ten injectable and eight oral DMT studies reported a mean 12-month adherence. For injectable DMTs, MPR $(\mathrm{N}=8)$ and PDC $(\mathrm{N}=3)$ adherence ranged, respectively, between $69.0 \%$ to $92.0 \%$, and $67.0 \%$ to $86.0 \%$. For two autoinjector studies, reported adherence was $97.0 \%$ and $98.0 \%$. For oral DMTs, MPR adherence $(\mathrm{N}=5)$ ranged between $68.0 \%$ and 92.0\%, PDC adherence $(\mathrm{N}=5)$ ranged between $73.0 \%$ and
83.0\%, and two studies measuring PC reported adherence as $89.0 \%$ and $99.0 \%$. One oral DMT adherence study utilizing MEMS cap reported $84.0 \%$ adherence.

Pooled MPR adherence for 12-month oral and injectable DMTs was, respectively, 91.0\% (95\% CI: 82.0\%-101.0\%) and 77.0\% (95\% CI: 66.0\%-87.0\%; Figure 2). Given the heterogeneity between studies (Cochran's Q: 766.16, $\mathrm{df}=5, \mathrm{P}<0.001$; I2: 99.3\%), we substantiated meta-analysis results using meta-regression(Table 2), which showed significantly higher mean adherence for oral compared to injectable DMTs $(\beta=-0.146 ; 95 \%$ CI: -0.263 to -0.029$)$. The doi plot points 
TABLE 2 Results from IVhet Meta-Regression Model Showing the Difference in Mean MPR Across Different Groups of Studies

\begin{tabular}{l|c|c|c|c|c|c}
\hline & Coef. & $\begin{array}{c}\text { Robust std. } \\
\text { err. }\end{array}$ & T & P>t & LCL & UCL \\
\hline Group 2 & -0.14 & 0.049 & -2.91 & 0.013 & -0.25 & -0.03 \\
\hline cons (Group-1) & 0.91 & 0.008 & 103.76 & 0.000 & 0.89 & 0.93 \\
\hline
\end{tabular}

Group-1, $n=6$; Group $-2, n=8$.

toward major asymmetry across studies (LFK index: -5.18; Supplementary Figure 2, available in online article), proposing study heterogeneity.

Over 24 months, injectable MPR adherence $(\mathrm{N}=5)$ ranged between $55.0 \%$ and $90.0 \%$, at 36 months injectable MPR $(\mathrm{N}=1)$ was $76.0 \%$, and autoinjector adherence $(\mathrm{N}=4)$ ranged from 91.0 to $95.0 \%$. There were no comparable oral DMT studies at 24 or 36 months.

\section{DISCONTINUATION RATES}

Discontinuation studies were defined by length: longer than a 30-day gap $(\mathrm{N}=3)$, at least a 60 -day gap $(\mathrm{N}=4)$, and longer than a 90-day gap $(\mathrm{N}=10)$. One study used temporary ( $<6$ months) and definitive discontinuation $(>12$ months). Eight studies did not provide a discontinuation definition (Supplementary Table 3).

There were four injectable studies with an observation period of less than 12 months, ranging between $5.0 \%$ and $26.2 \%$ discontinuation. One oral DMT study reported $28.0 \%$ discontinuation.

For 12-month injectable $(\mathrm{N}=10)$ and oral $(\mathrm{N}=7)$ DMT studies, overall mean discontinuation ranged, respectively, between $15.2 \%$ to $50.8 \%$ and $10.5 \%$ to $33.3 \%$. Meta-analysis for 12 -month studies ( $\mathrm{N}=10$ injectable and $\mathrm{N}=7$ oral DMTs) highlighted the presence of significant heterogeneity (I2 injectable: 99.5\%; I2 oral: 93.1\%) between studies, including within subgroups. There was no appreciable difference in 12-month mean discontinuation rates across groups (Figure 3, injectable: $27.0 \%$; $95 \%$ CI: 19.0\%-34.0\%; oral: 24.0\%; 95\% CI: 17.0\%-31.0\%).

Over a 24-month observation period, mean discontinuation for injectable DMTs $(\mathrm{N}=6)$ ranged between $6.3 \%$ and $57.0 \%$, and oral DMTs $(\mathrm{N}=7)$ ranged between $1.0 \%$ and $34.0 \%$. Only injectable DMT discontinuation data was available for over a 36-month observation period. The seven studies ranged between $9.7 \%$ and $53.6 \%$.

\section{Discussion}

This systematic review and metaanalysis synthesized and reported, across all measurement durations, on objectively measured self-administered injectable and oral DMT adherence and discontinuation rates in people with MS. To our knowledge, it is the first comparative review of injectable and oral DMT adherence and discontinuation rates. Suboptimal adherence and persistence were present for both injectable and oral DMTs. When compared with injectable DMTs and measured using mean adherence, meta-regression showed a $14 \%$ significant improvement in 12-month medication adherence for oral DMTs. The improvement contrasted with a 12-month oral and injectable adherence using a cut-off score of at least $80 \%$ to determine adherence, which showed no significant difference. Furthermore, there was no appreciable difference in 12-month discontinuation rates between oral and injectable DMTs.

While previous systematic reviews have examined injectable adherence or discontinuation ${ }^{8,15}$ or oral adherence and discontinuation rates ${ }^{20,21}$ in isolation, these results have enabled a comparison of adherence and discontinuation between these two administration modalities. In particular, the significant improvement in oral medication adherence proposes that administration modality may relate to medication adherence. Despite the improvement seen in oral DMT adherence, our review shows that suboptimal medication adherence and discontinuation of DMTs remains present for both injectable and oral DMTs.

\section{MEDICATION ADHERENCE}

Medication adherence rates varied vastly across studies. When reported utilizing a binary cut-off score of at least $80 \%$-across different measurement methods and calculations, such as claims, autoinjector and MEMS counts, MPR and PDC calculationsstudies reporting 12-month adherence ranged between $46.0 \%$ and $90.0 \%$ for injectable DMTs and between 53.0\% and $94.0 \%$ for oral DMTs. While there was no difference in adherence between oral and injectable DMTs when measured by a binary cut-off score of at least $80 \%$, there was a significant improvement in oral compared to injectable adherence for studies reporting 12-month mean adherence (91.0\% $[\mathrm{P}<0.001]$ vs $77.0 \%(\mathrm{P}=0.013)$, respectively). The improvement is likely due to reduced power associated with binary measurement. ${ }^{31}$ This highlights the importance of studies choosing adherence levels formulated from an appropriate rationale. While the $80 \%$ adherence cut-off score is most frequently used in the literature, it is arbitrarily applied. Indeed, adherence across drugs, diseases, and 


\section{FIGURE 3 Meta-Analysis of Discontinuation Rates}

\section{a. Oral DMTs}

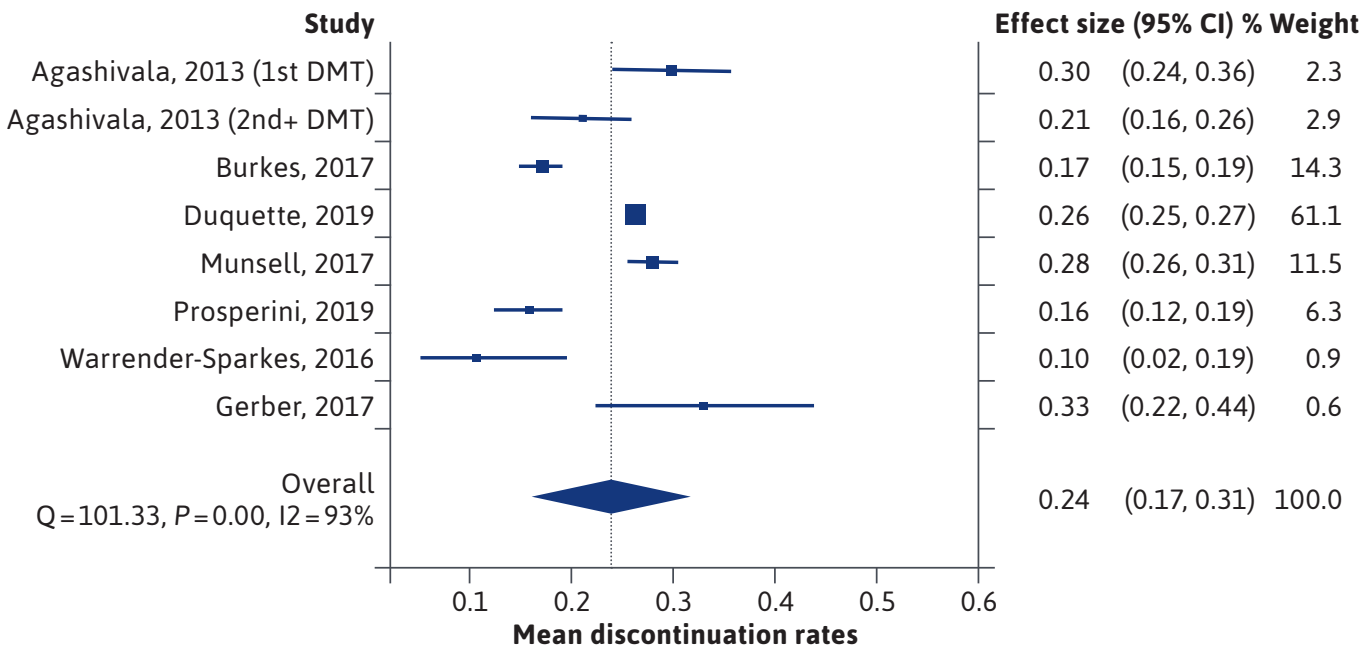

\section{b. Injectable DMTs}

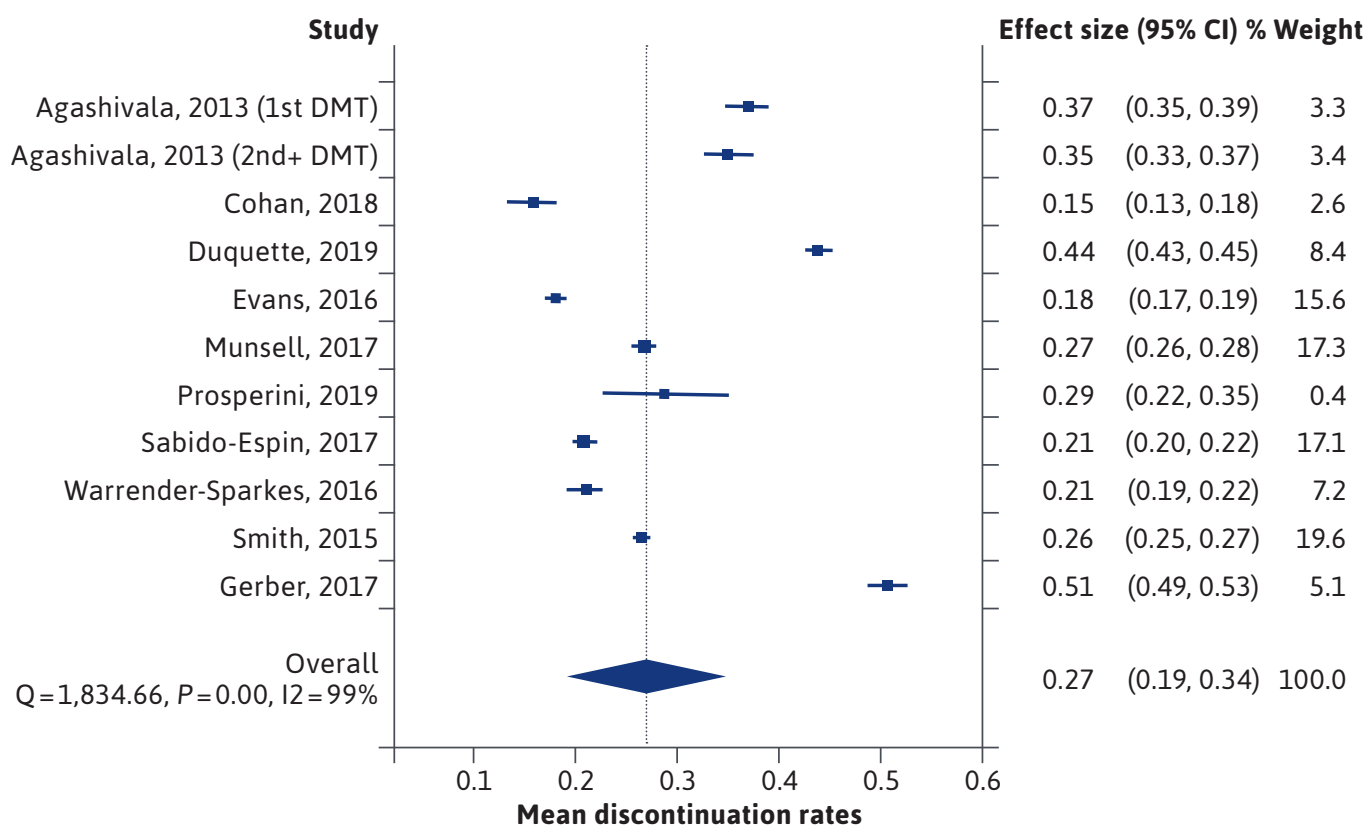

Notes: Pooled subgroup results are presented (overall pooled estimates not available). Weights are from Doi's IVHet Model. Model Effect $=$ discontinuation rates.

patient characteristics may differ ${ }^{32}$ Given that studies looking at adherence on outcome in MS most often use the $80 \%$ cut-off score to determine adherence, it is difficult to appreciate the clinical impact of the $14 \%$ statistically significant difference for health and economic outcomes. Across measurement types, 12-month mean adherence ranged between $67.0 \%$ to $98.0 \%$ for injectable and $68.0 \%$ to $99.0 \%$ for oral adherence. Beyond a 12-month duration, oral DMT data was 
limited, restricting any sort of valid comparative analysis.

Adherence rates for MEMS and autoinjectors were considerably higher than other measures. Use of autoinjectors may improve adherence, given that they may reduce the impact of injection-related issues, such as needle phobias and injection site reactions. ${ }^{18}$ However, higher results may be attributed to study parameters, such as study support or participant awareness of adherence observation, not afforded to participants measured via retrospective claims/ pharmacy refill data. ${ }^{33}$

Despite improved 12-month oral DMT adherence, in line with previous reviews, suboptimal adherence remains present for injectable and oral DMTs, illustrating the problematic issue of DMT adherence regardless of administration route. ${ }^{8,20,21}$ Thus, DMT adherence remains an issue requiring attention, given poorer adherence is linked to greater healthcare resource utilization and days of work lost. $^{34}$

\section{MEDICATION DISCONTINUATION}

As with DMT adherence, our review of discontinuation studies found vast differences in discontinuation rates. For 12-month discontinuation, injectable DMTs ranged between $15.2 \%$ and $50.8 \%$, while oral DMTs ranged between $10.5 \%$ and $33.3 \%$, yet there was no significant difference (pooled 12-month discontinuation: $27.0 \%$ and $24.0 \%$, respectively). For 24-months, injectable discontinuation ranged between $6.3 \%$ and $57.0 \%$, while oral studies ranged between $1.0 \%$ and $39.0 \%$.

Discontinuation rates varied depending on discontinuation definition. Shorter discontinuation definitions (ie, $>30$-day) resulted in higher discontinuation rates than longer discontinuation definitions (ie, $>90$-day), pointing to the incorrect categorization of 'having ceased their DMT' when people, may in fact, take 'medication holidays.' The error is highlighted by a long-term study ( $\mathrm{N}=721 ; \mathrm{M}=7.2$ years) that showed $57.4 \%$ of people with MS who discontinued a DMT recommenced at some point. ${ }^{35}$ The investigators reported that, when using a 90-day discontinuation definition, $62.6 \%$ of participants discontinued, compared with $26.4 \%$ of participants defined by a 12-month discontinuation. ${ }^{35}$ Reasons for treatment discontinuation and recommencement are not addressed in the study. Thus, varying discontinuation definitions and observation durations across studies may result in overestimating or underestimating rates of discontinuation, which presents issues with generalizability. This underscores the need for consistent study protocol use in DMT adherence and discontinuation studies, enabling comparative analysis.

Our findings are broadly comparable with a previous discontinuation review for injectable DMTs that found mean discontinuation rates ranged between $16-27 \%,{ }^{15}$ and the 12-month pooled oral discontinuation was similar to a recent review of $25.4 \% .^{21}$ Thus, the need remains to provide people with MS ongoing support, encouraging medication continuation regardless of DMT administration route. While this study focused on adherence differences between oral and injectable administration routes, it is acknowledged that DMTs will have different tolerability profiles that may impact adherence. Indeed, medication side effects are known to impact medication adherence and persistence. ${ }^{18}$ The current review excluded studies if they did not clearly account for switching to minimize the impact of methodological study bias on reported results. Despite the exclusion, discontinuation studies varied substantially in methodology.

\section{CLINICAL IMPLICATIONS}

The improvement in adherence of oral DMTs compared with injectable DMTs highlights a benefit of oral DMT administration that will translate into improved clinical outcomes for people with MS yet does not solve the problem of suboptimal medication adherence and persistent. Thus, people with MS continue to require support to develop strategies to take their medication as prescribed. This is underscored by an absence of improved medication persistence rates for oral DMTs, emphasizing the complexity surrounding medication adherence and persistence beyond self-injection administration issues.

People with MS may experience a range of risk factors for suboptimal medication adherence and discontinuation, including depression, cognitive impairment, complex medication administration regimes (polypharmacy or administration schedule requirements), and complex medical needs (eg, comorbid chronic illness). ${ }^{3,18}$ Awareness of these risk factors enables clinicians to be responsive to the potential impact on suboptimal adherence and, as required, to provide support and/or intervention referral. Appreciating issues of suboptimal adherence is also important to breakthrough disease activity or progression, which may trigger unwarranted DMT switching or escalation due to compliance related reduced efficacy. ${ }^{4,18}$

\section{RESEARCH IMPLICATIONS}

An evident outcome of this review, which reduces study comparability, is the inconsistencies between-study protocols, methodology, varying adherence thresholds, and discontinuation definitions. While the majority of studies classified participants as adherent using a binary threshold of at least 80, commonly accepted across adherences studies, some studies used incomparable cut scores, such as at least $85,{ }^{36}$ at least $75,{ }^{37}$ or categorical adherence with no explicit 
definition of adherence level. ${ }^{38}$ The significant difference between oral and injectable DMTs when assessing 12-month mean adherence, compared with 12-month binary adherence of at least $80 \%$-which was nonsignificantproposes a loss of power to detect medication adherence levels using binary adherence. ${ }^{31}$ Future studies would benefit from reporting mean 12-month adherence instead of, or in addition to, binary adherence results of at least $80 \%$.

The wide disparity across discontinuation definitions, ranging from a gap period of more than 30 days to more than 90 days and studies providing no discontinuation definition, limits confidence in the generalizability of study results. Higher discontinuation rates were reported for studies with shorter discontinuation definitions compared to longer gaps, pointing to a need for consistency of discontinuation definition, with longer discontinuation periods likely to provide greater accuracy. Future research would also benefit from identifying patterns of temporary discontinuations, or 'medication holidays,' to inform medication adherence interventions.

Given that switching to an alternative DMT in the event of breakthrough disease activity or adverse events is common in MS healthcare, and medication discontinuation research must account for this to avoid overestimating DMT discontinuation rates. DMT switch rates are shown to be between 19.7 to $30.0 \%{ }^{3,39,40}$ Fifteen studies were excluded from this review, as switching was not sufficiently considered. There is a need for methodological and reporting consistency for future studies to examine adherence and discontinuation behaviour in MS.

\section{STRENGTHS AND LIMITATIONS}

This review utilized a rigorous methodology, undertaking a thorough systematic literature search.
Separation of the adherence and discontinuation results by measurement type (ie, MPR and PDC), discontinuation definition (ie, 30-day, 60-day, and 90-day gap periods) and observation lengths (ie, $<8$ months to 36 months), and removal of studies that did not explicitly account for switching, were employed to reduce the chance of overestimating discontinuation. We utilized PROSPERO registration and PRISMA reporting guidelines to minimize reporting bias. The review contributes novel findings to the existing literature on adherence and discontinuation rates for both injectable and oral DMTs.

Results were limited by the large variation across methodology of the included studies, as discussed previously. Our focus on comparative results of 12-month adherence and discontinuation to identify differences in injectable and oral administration modalities attempts to minimize reporting bias and provide generalizable results. The large heterogeneity in the methodology of included studies meant we were only able to report ranges of individual studies for periods other than 12 months. This review examined between-group adherence and discontinuation rates for oral and injectable DMTs without consideration of within-group variability, which may be present at both the level of the individual person and DMT. ${ }^{15,20}$

\section{Conclusions}

In summary, this systematic review and meta-analysis compared adherence and discontinuation of oral and injectable DMTs for people with MS. There was a $14 \%$ improvement to 12-month mean oral DMT adherence, compared to injectable adherence, but no improvement to 12-month binary adherence using a cut-off score of at least $80 \%$. There was no difference in discontinuation rates between oral and injectable DMTs. Both oral and injectable DMTs suffer suboptimal medication adherence and discontinuation issues. Our review highlighted several methodological inconsistencies that limit betweenstudy comparisons, emphasizing the need for future adherence and discontinuation research to use consistent definitions, durations, and measures to improve the generalizability of findings and reliability of translatable implications into clinical practice.

\section{DISCLOSURES}

The authors did not receive any funding for this study. Mardan and Hussain have nothing to disclose. Grech reports grants from Merck Pharmaceutical, outside the submitted work. Allan reports grants received from Merck Pharmaceutical outside the submitted work. Allan holds advisory board and consulting positions with Merck and advisory board positions for Bristol Myers Squibb and Novartis, for which Monash Institute of Neurological Diseases receives consulting fees.

\section{REFERENCES}

1. Reich DS, Lucchinetti CF, Calabresi PA. Multiple sclerosis. New Engl J Med. 2018;378(2):169-80. doi:10.1056/ NEJMra1401483.

2. Wallin MT, Culpepper WJ, Nichols E, et al. Global, regional, and national burden of multiple sclerosis 1990-2016: a systematic analysis for the global burden of disease study 2016. Lancet Neurol. 2019;18(3):269-85. doi:10.1016/ S1474-4422(18)30443-5

3. Gajofatto A, Benedetti MD. Treatment strategies for multiple sclerosis: when to start, when to change, when to stop? World J Clin Cases. 2015;3(7):545-55. doi:10.12998/wjcc.v3.i7.545
4. Robertson D, Moreo N. Disease- modifying therapies in multiple sclerosis: overview and treatment considerations. Fed Pract. 2016;33(6):28-34. 
5. Giovannoni G, Comi G, Cook S, et al. A placebo-controlled trial of oral cladribine for relapsing multiple sclerosis. New Engl J Med. 2010;362(5):416-26. doi:10.1056/NEJMoa0902533

6. Torkildsen $\varnothing$, Myhr KM, Bø L. Disease-modifying treatments for multiple sclerosis - a review of approved medications. Eur J Neurol. 2016;23(Suppl 1(S1):18-27. doi:10.1111/ene.12883

7. World Health Organization. Adherence to long term therapies: evidence for action: World Health Organization; Published 2003. Accessed Febuary 24, 2019. https://apps.who.int/iris/bitstream/handle/10665/42682/9241545992. pdf

8. Menzin J, Caon C, Nichols C, White L, Friedman M, Pill M. Narrative review of the literature on adherence to diseasemodifying therapies among patients with multiple sclerosis. J Manag Care Spec Pharm. 2013;19(1 Supp A):S24-40. doi:10.18553/jmcp.2013.19.s1.S24

9. Duquette P, Yeung M, Mouallif S, Nakhaipour HR, Haddad P, Schecter R. A retrospective claims analysis: compliance and discontinuation rates among Canadian patients with multiple sclerosis treated with disease-modifying therapies. PLoS One. 2019;14(1):e0210417. doi:10.1371/ journal.pone.0210417

10.10.. Hansen K, Schussel K, Kieble M, et al. Adherence to disease modifying drugs among patients with multiple sclerosis in Germany: a retrospective cohort study. PLoS One. 2015;10(7):e0133279. doi:10.1371/journal.pone.0133279

11. Zettl UK, Bauer-Steinhusen U, Glaser T, et al. Adherence to long-term interferon beta-1b injection therapy in patients with multiple sclerosis using an electronic diary. Adv Ther. 2016;33(5):834-47. doi:10.1007/s12325-016-0325-6
12. Johnson KM, Zhou H, Lin F, Ko JJ, Herrera V. Real-world adherence and persistence to oral disease-modifying therapies in multiple sclerosis patients over 1 year. J Manag Care Spec Pharm. 2017;23(8):844-52. doi:10.18553/ jmcp.2017.23.8.844

13. Burks J, Marshall T, Ye X. Adherence to disease-modifying therapies and its impact on relapse, health resource utilization, and costs among patients with multiple sclerosis. Clinicoecon Outcomes Res. 2017;9:251-60. doi:10.2147/CEOR. $\mathrm{S} 130334$

14. Bruce JM, Hancock LM, Lynch SG. Objective adherence monitoring in multiple sclerosis: initial validation and association with self-report. Mult Scler. 2010;16(1):112-20. doi:10.1177/1352458509351897

15. Giovannoni G, Southam E, Waubant E. Systematic review of disease-modifying therapies to assess unmet needs in multiple sclerosis: tolerability and adherence. Mult Scler. 2012;16(1):932-46. doi:10.1177/1352458511433302

16. Cramer J, Roy A, Burrell A, et al. Medication compliance and persistence: terminology and definitions. Value Health. 2008;11(1):44-47. doi:10.1111/j.1524-4733.2007.00213.x

17. Raebel M, Schmittdiel J, Karter A, Konieczny J, Steiner J. Standardizing terminology and definitions of medication adherence and persistence in research employing electronic databases. Med Care. 2013;51(8 Suppl 3):11-21. doi:10.1097/ MLR.0b013e31829b1d2a

18. Patti F. Optimizing the benefit of multiple sclerosis therapy: the importance of treatment adherence. Patient Prefer Adherence. 2010;4:1-9. doi:10.2147/ppa. s8230
19. Lafata JE, Cerghet M, Dobie E, et al. Measuring adherence and persistence to disease-modifying agents among patients with relapsing remitting multiple sclerosis. J Am Pharm Assoc (2003). 2008;48(6):752-57. doi:10.1331/ JAPhA.2008.07116

20. Yoon EL, Cheong WL. Adherence to oral disease-modifying therapy in multiple sclerosis patients: a systematic review. Mult Scler Relat Disord. 2019;28:104-08. doi:10.1016/j.msard.2018.12.025

21. Nicholas JA, Edwards NC, Dellarole A, Grosso M, Phillips AL. Real-world adherence to, and persistence with, once- and twice-daily oral disease-modifying drugs in patients with multiple sclerosis: a systematic review and meta-analysis. BMC Neurol. 2020;20(1):281. doi:10.1186/ s12883-020-01830-0

22. US Food and Drug Administration. Drug approval package. FDA. Published June 11, 2017. Accessed Febuary 24, 2019. https://www.accessdata.fda.gov/ drugsatfda_docs/nda/pre96/ 103471s0000TOC.cfm

23. Doi SA, Barendregt JJ, Khan S, Thalib L, Williams GM. Advances in the meta-analysis of heterogeneous clinical trials I: the inverse variance heterogeneity model. Contemp Clin Trials. 2015;45(Pt A):130-38. doi:10.1016/j.cct.2015.05.009

24. Kelly GA, Kelly KS. Communitydeliverable exercise and depression in adults with arthritis: confirmatory evidence of a meta-analysis using the IVhet model. J Evid Based Med. 2018;11(1):51-55. doi:10.1111/jebm.12229

25. Furuya-Kanamori L, Barendregt JJ, Doi SA. A new improved graphical and quantitative method for detecting bias in meta-analysis. Int J Evid Based Healthc. 2018;16(4):195-203. doi:10.1097/ XEB.0000000000000141 
26. Doi SA, Barendregt JJ, Khan S, Thalib L, Williams GM. Advances in the meta-analysis of heterogeneous clinical trials II: the quality effects model. Contemp Clin Trials. 2015;45(Pt A):123-29. doi:10.1016/j.cct.2015.05.010

27. Downes MJ, Brennan ML, Williams HC, Dean RS. Development of a critical appraisal tool to assess the quality of cross-sectional studies (AXIS). BMJ Open. 2016;6(12):e011458. doi:10.1136/ bmjopen-2016-011458

28. Moher D, Liberati A, Tetzlaff J, Altman DG. Preferred reporting items for systematic reviews and meta-analysis: the PRISMA statement. BMJ. 2009;339:b2535. doi:10.1136/bmj.b2535

29. Landis JR, Koch GG. The measurement of observer agreement for categorical data. Biometrics. 1977;33(1):159-74.

30. Settle JR, Maloni HW, Bedra M, Finkelstein J, Zhan M, Wallin MT. Monitoring medication adherence in multiple sclerosis using a novel web-based tool: a pilot study. J Telemed Telecare. 2016;22(4):225-33. doi:10.1177/1357633X15597115

31. Austin PC, Brunner LJ. Inflation of the type I error rate when a continuous confounding variable is categorized in logistic regression analyses. Stat Med. 2004;23(7):1159-78. doi:10.1002/sim.1687

32. Baumgartner PC, Haynes RB, Hersberger KE, Arnet A. A systematic review of medication adherence thresholds dependent of clinical outcomes. Front Pharmacol. 2018;9(1290). doi:10.3389/ fphar.2018.01290

33. Blonde L, Khunti K, Harris SB, Meizinger C, Skolnik NS. Interpretation and impact of real-world clinical data for the practising clinician. Adv Ther. 2018;35(11):1763-74. doi:10.1007/ s12325-018-0805-y
34. Yermakov S, Davis M, Calnan M, et al. Impact of increasing adherence to disease-modifying therapies on healthcare resource utilization and direct medical and indirect work loss costs for patients with multiple sclerosis. J Med Econ. 2015;18(9):711-20. doi:10.3111/13696998.201 5.1044276

35. Melesse DY, Marrie RA, Blanchard JF, Yu BN, Evans C. Persistence to diseasemodifying therapies for multiple sclerosis in a Canadian cohort. Patient Prefer Adherence. 2017;11:1093-101. doi:10.2147/ PPA.S138263

36. Steinberg SC, Faris RJ, Chang CF, Chan A, Tankersley MA. Impact of adherence to interferons in the treatment of multiple sclerosis: a non-experimental, retrospective, cohort study. Clin Drug Investig. 2010;30(2):89-100. doi:10.2165/11533330-000000000-00000

37. Bayas A, Ouallet JC, Kallmann B, Hupperts R, Fulda U, Marhardt K. Adherence to, and effectiveness of, subcutaneous interferon beta-1a administered by RebiSmart(R) in patients with relapsing multiple sclerosis: results of the 1-year, observational SMART study. Expert Opin Drug Deliv. 2015;12(8):1239-50. doi:10.1517/ 17425247.2015.1057567

38. Zecca C, Disanto G, Muhl S, Gobbi C. Subjective patient-reported versus objective adherence to subcutaneous interferon beta-1a in multiple sclerosis using RebiSmart(R): the CORE study. BMC Neurol. 2017;17(1):171. doi:10.1186/ s12883-017-0952-9

39. Bonafede MM, Johnson BH, Wenten M, Watson C. Treatment patterns in diseasemodifying therapy for patients with multiple sclerosis in the United States. Clin Ther. 2013;35(10):1501-12. doi:10.1016/ j.clinthera.2013.07.330
40. Degil Esposti L, Piccinni C, Sangiorgi D, et al. Changes in first-line injectable disease-modifying therapy for multiple sclerosis: predictors of non-adherence, switching, discontinuation, and interruption of drugs. Neurol Sci. 2017;38(4):589-94. doi:10.1007/ s10072-016-2806-4

41. Agashivala N, Wu N, Abouzaid S, et al. Compliance to fingolimod and other disease modifying treatments in multiple sclerosis patients, a retrospective cohort study. BMC Neurol. 2013;13:138. doi:10.1186/1471-2377-13-138

42. Aungst A, Casady L, Dixon C, et al. Assessing barriers to adherence with the use of dimethyl fumarate in multiple sclerosis. Clin Drug Investig. 2020;40(1):73-81. doi:10.1007/s40261-019-00866-6

43. Braune S, Lang M, Bergmann A. Efficacy of fingolimod is superior to injectable disease modifying therapies in second-line therapy of relapsing remitting multiple sclerosis. J Neurol. 2016;263(2):327-33. doi:10.1007/ s00415-015-7970-6

44. Cerghet M, Dobie E, Lafata JE, et al. Adherence to disease-modifying agents and association with quality of life among patients with relapsing-remitting multiple sclerosis. Int J MS Care. 2010;12(2):51-58. doi:10.7224/1537-2073-12.2.51

45. Cohan S, Smoot K, Kresa-Reahl K, et al. Outcomes of stable multiple sclerosis patients staying on initial interferon beta therapy versus switching to another interferon beta therapy: a US claims database study. Adv Ther. 2018;35(11):1894-904. doi:10.1007/s12325-018-0799-5

46. Cohen BA, Coyle PK, Leist T, Oleen-Burkey MA, Schwartz M, Zwibel H. Therapy optimization in multiple sclerosis: a cohort study of therapy adherence and risk of relapse. Mult Scler Relat Disord. 2015;4(1):75-82. doi:10.1016/j. msard.2014.09.214 
47. Condé S, Moisset X, Pereira B, et al. Dimethyl fumarate and teriflunomide for multiple sclerosis in a real-life setting: a French retrospective cohort study. Eur J Neurol. 2019;26(3):460-67. doi:10.1111/ ene.13839

48. D'Amico E, Zanghì A, Sciandra M, et al. Discontinuation of teriflunomide and dimethyl fumarate in a large Italian multicentre population: a 24-month real-world experience. J Neurol. 2019;266(2):411-16. doi:10.1007/s00415-018-9144-9

49. Defer G, de Seze J, Bouee S, et al. Outcomes and treatment management of a French cohort suffering from multiple sclerosis: a retrospective epidemiological study. Mult Scler Relat Disord. 2018;25:276-81. doi:10.1016/j. msard.2018.08.004

50. Deftereos SN, Koutlas E, Koutsouraki E, et al. Seasonal adherence to, and effectiveness of, subcutaneous interferon beta-1a administered by RebiSmart(R) in patients with relapsing multiple sclerosis: results of the 1-year, observational GEPAT-SMART study. BMC Neurol. 2018;18(1):186. doi:10.1186/ s12883-018-1179-0

51. Devonshire VA, Feinstein A, Moriarty P. Adherence to interferon beta-1a therapy using an electronic self-injector in multiple sclerosis: a multicentre, single-arm, observational, phase IV study. BMC Res Notes. 2016;9:148.

doi:10.1186/s13104-016-1948-z

52. Eriksson I, Cars T, Piehl F, Malmström RE, Wettermark B, von Euler M. Persistence with dimethyl fumarate in relapsing-remitting multiple sclerosis: a population-based cohort study. Eur J Clin Pharmacol. 2018;74(2):219-26. doi:10.1007/ s00228-017-2366-4
53. Ernst FR, Barr P, Elmor R, Wong SL. Relapse outcomes, safety, and treatment patterns in patients diagnosed with relapsing-remitting multiple sclerosis and initiated on subcutaneous interferon beta-1a or dimethyl fumarate: a real-world study. Curr Med Res Opin. 2017;33(12):2099-106. doi:10.1080/0300799 5.2017 .1380616

54. Evans C, Marrie RA, Zhu F, et al. Adherence to disease-modifying therapies for multiple sclerosis and subsequent hospitalizations. Pharmacoepidemoil Drug Saf. 2017;26(6):702-11. doi:10.1002/pds.4207

55. Evans C, Marrie RA, Zhu F, et al. Adherence and persistence to drug therapies for multiple sclerosis: a population-based study. Mult Scler Relat Disord. 2016;8:78-85. doi:10.1016/j. msard.2016.05.006

56. Evans C, Tam J, Kingwell E, Oger J, Tremlett H. Long-term persistence with the immunomodulatory drugs for multiple sclerosis: a retrospective database study. Clin Ther. 2012;34(2):341-50. doi:10.1016/j. clinthera.2012.01.006

57. Fernández O, Arroyo R, Martinez-Yélamos S, et al. Long-term adherence to IFN beta-1a treatment when using RebiSmart(R) device in patients with relapsing-remitting multiple sclerosis. PLoS One. 2016;11(8):e0160313. doi:10.1371/ journal.pone.0160313

58. Fernández-Fournier M, Tallón-Barranco A, Chamorro B, Martínez-Sánchez P, Peurtas I. Differential glatiramer acetate treatment persistence in treatment-naive patients compared to patients previously treated with interferon. BMC Neurol. 2015;15:141. doi:10.1186/s12883-015-0399-9

59. Gerber B, Cowling T, Chen G, Teung M, Duquette P, Haddad P. The impact of treatment adherence on clinical and economic outcomes in multiple sclerosis: real world evidence from Alberta, Canada. Mult Scler Relat Disord. 2017;18:218-24. doi:10.1016/j.msard.2017.10.001
60. Jones JL, Scheidt DJ, Kaushal RS, Carroll CA. Assessing the role of patient support services on adherence rates in patients using glatiramer acetate for relapsing-remitting multiple sclerosis. J Med Econ. 2013;16(2):213-20. doi:10.3111/1 3696998.2012 .744316

61. Kleinman NL, Beren IA, Rajagopalan K, Brook RA. Medication adherence with disease modifying treatments for multiple sclerosis among US employees. J Med Econ. 2010;13(4):633-40. doi:10.3111/136969 98.2010 .527588

62. Kleiter I, Lang M, Jeske J, Norenberg C, Stollfub B, Schürks M. Adherence, satisfaction and functional health status among patients with multiple sclerosis using the BETACONNECT(R) autoinjector: a prospective observational cohort study. BMC Neurol. 2017;17(1):174. doi:10.1186/ s12883-017-0953-8

63. Kozma CM, Phillips AL, Meletiche DM. Use of an early disease-modifying drug adherence measure to predict future adherence in patients with multiple sclerosis. J Manag Care Spec Pharm. 2014;20(8):800-07. doi:10.18553/ jmcp.2014.20.8.800

64. Krol M, de Voer G, Osowski U. Patient adherence to subcutaneous IFN beta-1a injections using the RebiSmart(R) injection device: a retrospective real-world study among Dutch and German Patients with multiple sclerosis. Patient Prefer Adherence. 2017;11:1189-96. doi:10.2147/ PPA.S130985

65. Lebrun-Frenay C, Moulignier A, Pierrot-Deseilligny $\mathrm{C}$, et al. Five-year outcome in the copaxone observatory: a nationwide cohort of patients with multiple sclerosis starting treatment with glatiramer acetate in France. J Neurol. 2019;266(4):888-901. doi:10.1007/ s00415-019-09211-5 
66. Lugaresi A, Florio C, Brescia-Morra V, et al. Patient adherence and tolerability of self-adminstered interferon beta-1a using an electronic autoinjection device: a multicentre, open-label, phase IV study. BMC Neurol. 2012;12:7. doi:10.1186/1471-2377-12-7

67. McKay KA, Evans C, Fisk JD, et al. Disease-modifying therapies and adherence in multiple sclerosis: comparing patient self-report with pharmacy records. Neuroepidemiology. 2017;48(34):124-130. doi:10.1159/000477771

68. Moccia M, Pallandino R, Carotenuto A, et al. Predictors of long-term interferon discontinuation in newly diagnosed relapsing multiple sclerosis. Mult Scler Relat Disord. 2016;10:90-96. doi:10.1016/j. msard.2016.09.011

69. Moccia M, Palladino R, Russo C, et al. How many injections did you miss last month? A simple question to predict interferon beta-1a adherence in multiple sclerosis. Expert Opin Drug Deliv. 2015;12(12):1829-35. doi:10.1517/17425247.20 15.1078789

70. Munsell M, Frean M, Menzin J, Phillips AL. An evaluation of adherence in patients with multiple sclerosis newly initiating treatment with a self-injectable or an oral disease-modifying drug. Patient Prefer Adherence. 2016;11:55-62. doi:10.2147/PPA.S118107

71. Oleen-Burkey MA, Dor A, Castelli-Haley J, Lage MJ. The relationship between alternative medication possession ratio thresholds and outcomes: evidence from the use of glatiramer acetate. J Med Econ. 2011;14(6):739-47. doi:10.3 111/13696998.2011.618517

72. Paolicelli D, Cocco E, Di Lecce V, et al. Exploratory analysis of predictors of patient adherence to subcutaneous interferon beta-1a in multiple sclerosis: TRACER study. Expert Opin Drug Deliv. 2016;13(6):799-805. doi:10.1517/17425247.20 16.1158161
73. Pedersen ED, Stenager E, Vadgaard JL, et al. Adherence to subcutaneous interferon beta-1a treatment using an electronic injection device: a prospective open-label Scandinavian noninterventional study (the ScanSmart study). Patient Prefer Adherence. 2018;12:569-75. doi:10.2147/PPA.S154417

74. Prosperini L, Cortese A, Lucchini M, et al. Exit strategies for "needle fatigue" in multiple sclerosis: a propensity score-matched comparison study. J Neurol. 2020;267(3):694-702. doi:10.1007/ s00415-019-09625-1

75. Sabidó-Espin M, Munschauer R. Reasons for discontinuation of subcutaneous interferon beta-1a three times a week among patients multiple sclerosis: a real-world cohort study. BMC Neurol. 2017;17(1):57. doi:10.1186/s12883-017-0831-4

76. Sanchirico M, Caldwell-Tarr A, Midumby P, Hashemi, Dufour R.

Treatment patterns, healthcare resource utilization, and costs among medicare patients with multiple sclerosis in relation to disease-modifying therapy and corticosteroid treatment. Neurol Ther. 2019;8(1):121-33. doi:10.1007/ s40120-018-0123-y

77. Schreiber K, Kant M, Pfleger C, et al. High treatment adherence, satisfaction, motivation, and health-related quality of life with fingolimod in patients with relapsing-remitting multiple sclerosis - results from a 24-month, multicenter, open-label Danish study. Patient Prefer Adherence. 2018;12:1139-50. doi:10.2147/ PPA.S166278

78. Shao H, Stoecker C, Monnette AM, Shi L. Cost sharing of disease-modifying treatments (DMTs) as policy lever to improve DMTs' access in multiple sclerosis. Value Health. 2018;21(9):1083-89. doi:10.1016/j.jval.2017.10.025
79. Smith MY, Sabidó-Espin M, Trochanov A, et al. Postmarketing safety profile of subcutaneous interferon beta1a given 3 times weekly: a retrospective administrative claims analysis. J Manag Care Spec Pharm. 2015;21(8):650-60. doi:10.18553/jmcp.2015.21.8.650

80. Edo Solsona MD, Monet Boquet EM, Casanova Estruch BC, Poveda Andrés JL. Impact of adherence on subcutaneous interferon beta-1a effectiveness administered by RemiSmart(R) in patients with multiple sclerosis. Patient Prefer Adherence. 2017;11:415-21. doi:10.2147/PPA. S127508

81. Stockl KM, Shin JS, Gong S, Harada AS, Solow BK, Lew HC. Improving patient selfmanagement of multiple sclerosis through a disease therapy management program. Am J Manag Care. 2010;16(2):139-44.

82. Tan H, Cai Q, Agarwal S, Stephenson JJ, Kamat S. Impact of adherence to disease-modifying therapies on clinical and economic outcomes among patients with multiple sclerosis. Adv Ther. 2011;28(1):51-61. doi:10.1007/ s12325-010-0093-7

83. Vieira MC, Conway D, Cox GM, et al. Time to treatment failure following initiation of fingolimod versus teriflunomide for multiple sclerosis: a retrospective US claims study. Curr Med Res Opin. 2020;36(2):261-70. doi:10.1080/03007995.2 019.1690440

84. Warrender-Sparkes M, Spelman T, Izquierdo $\mathrm{G}$, et al. The effect of immunomodulatory therapy on treatment uptake and persistence in multiple sclerosis. Mult Scler. 2016;22(4):520-32. doi:10.1177/1352458515594041

85. Williams MJ, Johnson K, Trenz HM, et al. Adherence, persistence, and discontinuation among Hispanic and African American patients with multiple sclerosis treated with fingolimod or glatiramer acetate. Curr Med Res Opin. 2018;34(1):107-15. doi:10.1080/03007995.2017.1374937 
86. Zhang T, Kingwell E, Zhu F, et al. Effect of adherence to the first-generation injectable immunomodulatory drugs on disability accumulation in multiple sclerosis: a longitudinal cohort study. BMJ Open. 2017;7(9):e018612. doi:10.1136/ bmjopen-2017-018612

87. Zhornitsky S, Greenfield J, Koch MW, et al. Long-term persistence with injectable therapy in relapsing-remitting multiple sclerosis: an 18-year observational cohort study. PLoS One. 2015;10(4):e0123824. doi:10.1371/journal. pone. 0123824
88. Zimmer A, Coslovsky M, Abraham I, Décard BF. Adherence to fingolimod in multiple sclerosis: an investigatorinitiated, prospective, observational, single-center cohort study. Patient Prefer Adherence. 2017;11:1815-1830. doi:10.2147/ PPA.S140293
89. Cramer JA, Roy A, Burrell A, et al. Medication Compliance and Persistence: Terminology and Definitions. Value Health. 2008;11(1):44-47. doi:10.1111/j.1524-4733.2007.00213.x 